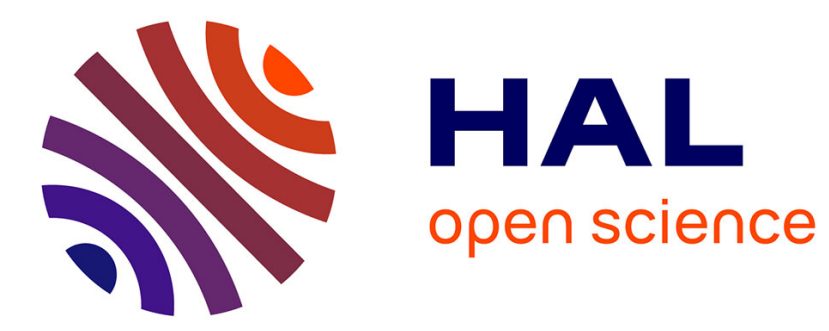

\title{
TomoFlow: Analysis of continuous conformational variability of macromolecules in cryogenic subtomograms based on 3D dense optical flow
}

Mohamad Harastani, Mikhail Eltsov, Amélie Leforestier, Slavica Jonic

\section{- To cite this version:}

Mohamad Harastani, Mikhail Eltsov, Amélie Leforestier, Slavica Jonic. TomoFlow: Analysis of continuous conformational variability of macromolecules in cryogenic subtomograms based on 3D dense optical flow. Journal of Molecular Biology, 2022, 434 (2), pp.167381. 10.1016/j.jmb.2021.167381 . hal-03452809

\author{
HAL Id: hal-03452809 \\ https://hal.science/hal-03452809
}

Submitted on 27 Nov 2021

HAL is a multi-disciplinary open access archive for the deposit and dissemination of scientific research documents, whether they are published or not. The documents may come from teaching and research institutions in France or abroad, or from public or private research centers.
L'archive ouverte pluridisciplinaire HAL, est destinée au dépôt et à la diffusion de documents scientifiques de niveau recherche, publiés ou non, émanant des établissements d'enseignement et de recherche français ou étrangers, des laboratoires publics ou privés. 
This is the author's version of an article accepted for publication in Journal of Molecular Biology, doi: https://doi.org/10.1016/j.jmb.2021.167381

\title{
TomoFlow: Analysis of continuous conformational variability of macromolecules in cryogenic subtomograms based on 3D dense optical flow
}

\author{
Mohamad Harastani ${ }^{1,2}$, Mikhail Eltsov ${ }^{3}$, Amélie Leforestier $^{2}$, and Slavica Jonic ${ }^{1,{ }^{*}}$ \\ ${ }^{1}$ IMPMC - UMR 7590 CNRS, Sorbonne Université, Muséum National d'Histoire Naturelle, Paris, France \\ ${ }^{2}$ Laboratoire de Physique des Solides (LPS), UMR 8502 CNRS, Université Paris-Saclay, Orsay, France \\ ${ }^{3}$ Department of Integrated Structural Biology, Institute of Genetics and Molecular and Cellular Biology, Illkirch, France
}

Contact details of the corresponding author:

Dr. Slavica Jonić

Sorbonne Université

IMPMC - CNRS UMR 7590, CC 115

4 Place Jussieu, 75005 Paris, France

Tel: +3314427 2205

Fax: +33144273785

E-mail: slavica.jonic@upmc.fr

Running title: TomoFlow 
Abstract: Cryogenic Electron Tomography (cryo-ET) allows structural and dynamics studies of macromolecules in situ. Averaging different copies of imaged macromolecules is commonly used to obtain their structure at higher resolution and discrete classification to analyze their dynamics. Instrumental and data processing developments are progressively equipping cryo-ET studies with the ability to escape the trap of classification into a complete continuous conformational variability analysis. In this work, we propose TomoFlow, a method for analyzing macromolecular continuous conformational variability in cryo-ET subtomograms based on a three-dimensional dense optical flow (OF) approach. The resultant lower-dimensional conformational space allows generating movies of macromolecular motion and obtaining subtomogram averages by grouping conformationally similar subtomograms. The animations and the subtomogram group averages reveal accurate trajectories of macromolecular motion based on a novel mathematical model that makes use of OF properties. This paper describes TomoFlow with tests on simulated datasets generated using different techniques, namely Normal Mode Analysis and Molecular Dynamics Simulation. It also shows an application of TomoFlow on a dataset of nucleosomes in situ, which provided promising results coherent with previous findings using the same dataset but without imposing any prior knowledge on the analysis of the conformational variability. The method is discussed with its potential uses and limitations.

Keywords: Cryogenic electron tomography (cryo-ET), continuous conformational variability analysis, optical flow, nucleosomes in situ. 


\section{Introduction}

Cryogenic electron tomography (cryo-ET) is an evolving structural biology technique that allows three-dimensional (3D) visualization and modeling of macromolecules in vitro and in situ. It allows obtaining volumetric scenes (so-called tomograms) of both thin layers of vitreous ice accommodating purified macromolecules and vitrified cell sections containing macromolecules in their native cellular environment. Cellular tomography is performed via several steps, including specimen vitrification [1, 2], obtaining thin sections or lamellae from the vitrified specimen $[3,4]$, projecting the thin sections from different tilting angles using a cryogenic electron microscope to get a set of 2D images (so-called tilt series), and reconstructing a tomogram from the tilt series [5].

Obtaining 3D models of macromolecules is essential for understanding their working mechanisms. However, macromolecules continuously change their shapes (conformations), which implies that one should ideally find the ensemble of 3D models describing the continuous conformational variability (conformational landscape) of the macromolecule under study in order to achieve a good understanding of its functions [6-9]. In the last decade, there has been a growing interest in methods development for continuous conformational variability analysis by cryogenic electron microscopy (cryo-EM) of purified complexes, better known as single particle analysis [6-16], and only recently by cryo-ET [17, 18].

Cryo-ET data suffers from several challenges in processing and interpretation, including but not limited to i) low signal-to-noise ratio (SNR) due to the low electron dose that is used to obtain tilt series in order to keep minimum radiation damage of the fragile biological specimens and ii) the so-called missing-wedge (MW) artifacts due to the limitation in the tilting range, commonly $\left[-60^{\circ}\right.$ to $\left.60^{\circ}\right]$. $\mathrm{MW}$ artifacts are data anisotropies in real space that are due to the corresponding empty wedge-shaped regions in Fourier space.

A typical tomogram can contain hundreds of copies of a particular macromolecule at different locations, orientations, and conformations. These copies are identified and isolated in independent subvolumes commonly referred to as subtomograms [19]. Ideally, identifying and analyzing subtomograms can lead to deciphering different conformations of the macromolecule. However, due to the data challenges, interpreting individual subtomograms is a difficult task. As a compromise, i) a 3D structural determination technique has emerged based on iterative rigid-body alignment and averaging of 
subtomograms (StA) [20], and ii) conformational variability is usually addressed via classification into discrete classes of structures.

Subtomogram classification methods can be divided into two families. The first family performs classification during StA, commonly via maximum-likelihood estimation and multi-reference alignment $[21,22]$. The second family performs classification on an aligned dataset (posterior to StA) [23, 24], typically based on the data covariance matrix of pairwise constrained cross-correlations between the subtomograms. There are significant challenges that are witnessed when classifying a dataset exhibiting continuous variability, including but not limited to i) the higher the number of classes requested, the lower the number of particles in each class, ii) redundant or empty classes are commonly observed, iii) stability of classes is not guaranteed, and iv) different algorithms may yield different results.

Nevertheless, obtaining the proper signal for analyzing individual macromolecules in cells is pursued for nearly five decades $[25,26]$. In recent years, more attention was drawn towards MW correction and denoising that allow analyzing individual subtomograms $[27,28]$. Only very recently, individual subtomogram analysis has been proposed for an exhaustive analysis of continuous conformational variability of macromolecules (HEMNMA-3D method [18]). To find the correct macromolecular conformation in a given individual subtomogram, HEMNMA-3D performs flexible fitting of a reference model (an atomic structure or a pseudoatomic structure from a density map such as a subtomogram average) into the subtomogram using normal modes (simulated directions of atomic or pseudoatomic motions). Simultaneously with searching for the correct macromolecular conformation, it searches for its correct rigid-body alignment with the subtomogram. Then, it projects the conformational parameters obtained by flexible fitting (normal mode amplitudes) for the given set of subtomograms onto a lowerdimensional space (referred to as conformational space) via a dimensionality reduction technique (e.g., Principal Component Analysis (PCA) [29]). However, HEMNMA-3D is computationally expensive and, thus, it does not use the entire set of available normal modes but requires choosing a smaller subset of potentially most relevant modes. Although the guidelines on how to choose the subset of normal modes have been provided, this task remains difficult in general. Besides, as it uses normal modes for the subtomogram analysis, HEMNMA-3D relies on prior knowledge of possible motions of the macromolecule. 
In this work, we propose TomoFlow, a method for an exhaustive analysis of continuous conformational variability in a given cryo-ET dataset, which uses no prior knowledge of possible motions of the macromolecules and analyses individual subtomograms based on 3D dense OF. Optical flow (OF) is a computer vision algorithm that is used in a wide range of applications. The concept of OF was proposed in the 1940s, and throughout the years, it has undergone massive improvements in the accuracy and computational efficiency of the algorithm until our days [30]. Dense OF takes two images of a similar scene as input and finds a vector per pixel that relates the pixels of one image with the pixels of the other image. The directions and amplitudes of these vectors give rise to a motion field that can be used for rigid and elastic alignment between the images. Some of the recent OF algorithms are very loyal to the data and can work with low SNR. OF drew the attention of the cryo-EM community, and several OF-based methods for cryo-EM data processing were proposed, including movie refinement [31] and mapping images of different conformations to 3D classes from single particle analysis [32].

In the TomoFlow method, the OFs are calculated between a reference (e.g., subtomogram average) and each of the subtomograms after MW correction and rigid-body alignment. Then, the OFs are used to refine the rigid-body alignment considering the conformational variability iteratively. Afterward, the OFs are collectively analyzed by constructing their Gram matrix and applying a dimensionality reduction technique (e.g., PCA). The resultant lower-dimensional space (referred to as conformational space) allows selective grouping of conformationally similar subtomograms and computing subtomogram averages from these groups, and it allows generating movies of macromolecular motion by animating the reference traversing data distribution manifolds in the conformational space (dense regions in this space). The animations and subtomogram group averages can reveal accurate trajectories of the macromolecular motion based on a novel derived mathematical model that makes use of the Gram matrix and OF properties.

This paper describes TomoFlow with tests on simulated datasets generated using different techniques, namely Normal Mode Analysis (NMA) and Molecular Dynamics (MD) simulation. It also shows an application on a dataset for nucleosomes in situ. Nucleosomes, fundamental organizers of eukaryotic genome into chromatin, are recognized as a family of conformations due to their complex intrinsic dynamics, post translational modifications, incorporation of histone variants, and interaction with remodelers, transcription factors, etc. [33]. Interestingly, continuous conformational changes of 
nucleosomes have been predicted $[34,35]$ as well as detected in vitro [36-38] and in situ $[17,18]$. In particular, in situ cryo-ET recently enabled capturing nucleosomes directly in their functional environment $[17,18,39]$, which opens new horizons to explore their continuous conformational space in relation to regulation of gene expression, DNA repair and replication. TomoFlow reveals a combination of breathing and gaping movements of nucleosomes, in good agreement with previous findings $[17,18]$. Lastly, the TomoFlow method is discussed with its potential uses and limitations.

\section{Methods}

This section presents the proposed method, TomoFlow, for macromolecular continuous conformational variability analysis in cryo-ET subtomograms using 3D dense OF. It first introduces the method's general scheme and objectives and then walks the reader through its building blocks with the necessary mathematical derivations and theoretical background.

TomoFlow (shown in Figure 1) analyzes the conformational variability in subtomograms after MWcorrection and rigid-body alignment. It performs OF-based matching of the subtomograms with an input reference (e.g., global subtomogram average) in the presence of a mask of the region of interest. Then, it collectively analyzes the resultant OFs between the input reference and each of the subtomograms by finding their Gram matrix and mapping it to a lower-dimensional space called the space of conformations (e.g., via PCA). In the conformational space, each point corresponds to a subtomogram, and close points correspond to subtomograms containing similar conformations. Accordingly, the conformational space is interactively processed by i) grouping close and dense regions and averaging the corresponding subtomograms to obtain subtomogram averages at different conformations (without classification), and ii) generating movie animations on the input reference while it fits curves in the conformational space following data distribution manifolds, i.e., animating the input reference to show the motion following the dense regions in the space. 


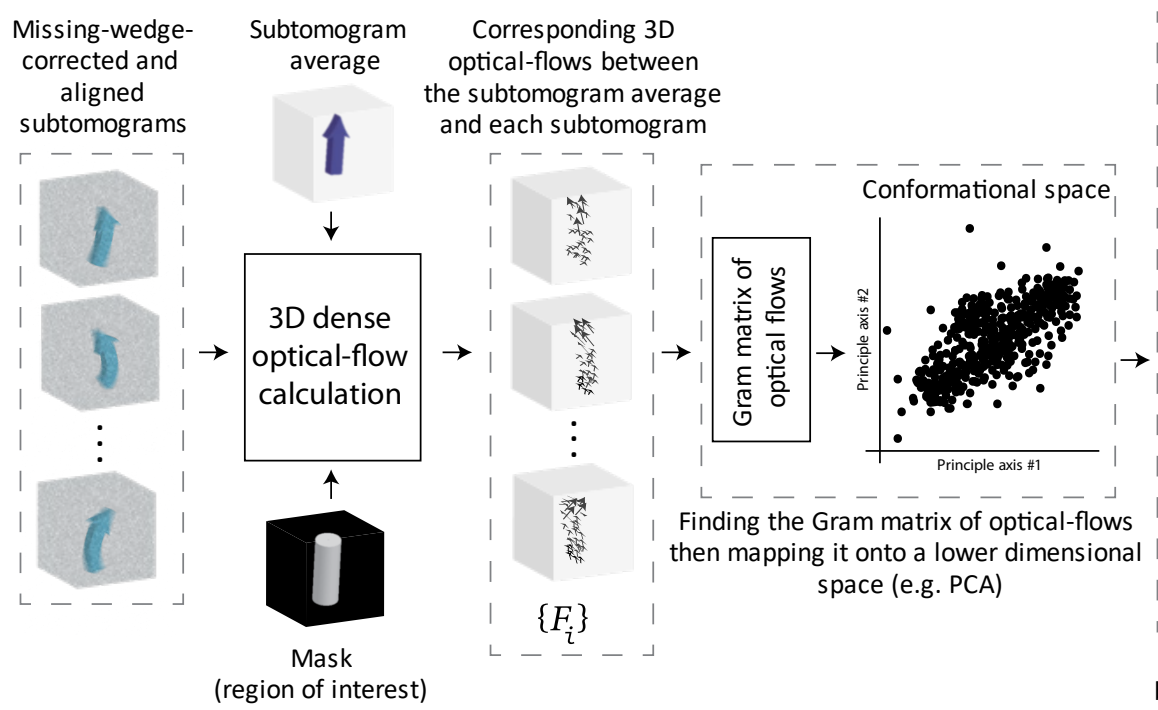

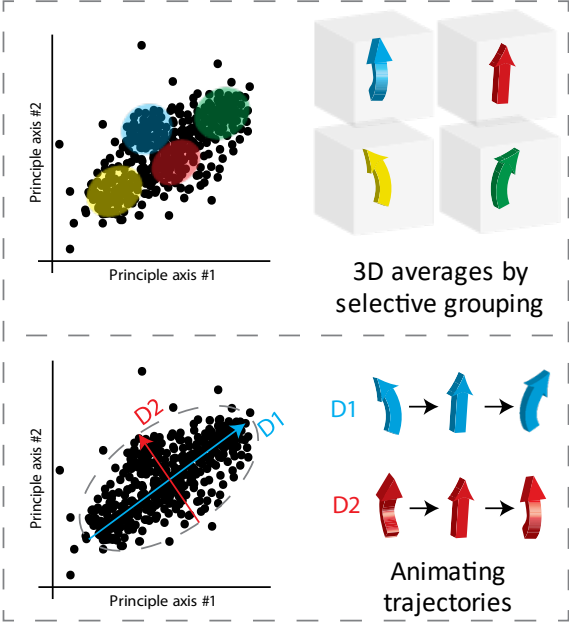

Interacively processing the conformational space by selective 3D averages and animating trajectories

Figure 1 Proposed pipeline for analyzing conformational variability in a set of subtomograms using 3D dense optical flows between a reference (here, subtomogram average) and each of the subtomograms.

\subsection{Employment of 3D dense OF for elastic and rigid-body matching of subtomograms}

This subsection introduces the working principles of 3D dense OF. It then explains how OF can be employed for 3D elastic and rigid-body matching, allowing subtomogram rigid-body alignment refinement and continuous conformational variability analysis.

3D dense OF is an algorithm that aims at finding the voxel-to-voxel correspondence between two volumetric images. OF calculations depend on two principles; the first principle is the brightness consistency, which means that the gray-level values (i.e., the brightness) of the corresponding voxels in the two input volumes are similar. To find the relationship between the voxels of two volumes $I$ and $H$, we assume that for a voxel $(x, y, z)$ in $H$, a voxel in $I$ with similar brightness can be found at some distance $(u, v, w)$ on $x, y$, and $z$ axis respectively:

$$
H(x, y, z) \approx I(x+u, y+v, z+w)
$$

The second principle is that the distance $(u, v, w)$ is small and that a limited number of terms (e.g., one term) of a Taylor expansion of the right-hand term of equation (1) is enough to describe the motion:

$$
I(x+u, y+v, z+w) \approx I(x, y, z)+\frac{d I}{d x} u+\frac{d I}{d y} v+\frac{d I}{d z} w
$$


Dense OF between $I$ and $H$ can be defined as the set of magnitudes $(u, v, w)$ for all $(x, y, z)$ between $I$ and $H$ to satisfy (1-2).

The two principles above established a practical computational background for OF over the years. Still, OF has suffered limitations in its functionality when the corresponding pixels (or voxels) in the two input images (or volumes) do not have the same brightness or are significantly distanced, which rendered OF sensitive to noise and only accounting for small displacements [30]. The 2D OF method of Farnebäck [40], which is a more recent approach, deals with these issues by combining two features. The first is enforcing local smoothness of the OF (close pixels move in the same direction) by approximating a neighborhood of each pixel in each of two given images with a polynomial (the coefficients of the local polynomial are estimated from a weighted least squares fit to the signal values in the neighborhood) and by integrating information about the displacement field between the two images over a neighborhood of each pixel. The data approximation by local polynomials is similar to local data smoothing and the displacement field integration over a pixel neighborhood is similar to local OF smoothing. It should also be noted that this method is not based on calculating image gradients (in equation (2)), but it finds a solution of a set of linear algebraic equations (the displacement of a pixel is calculated by directly evaluating matrices expressed in terms of the polynomial coefficients over a neighborhood of the pixel) and this solution is generally unique except in the case when the neighborhood is exposed to the aperture problem [40]). The aperture problem refers to the fact that when a moving object is viewed through a limited-size aperture, the direction of motion of a local feature or a region of the object may be ambiguous. In general, this problem is relevant to rigid objects with straight-line edges or flat regions (e.g., for a moving rectangle, motion of an edge in the direction perpendicular to that edge can be determined unambiguously, but motion of the edge along itself and motion of the inner flat region of the rectangle cannot be determined unambiguously). This problem is less relevant to cryo-EM and cryo-ET of biological macromolecules, which are generally flexible with curved edges and without flat regions. The second important feature of the method of Farnebäck [40] is calculating OF iteratively and over multiple scales of the input images (image pyramids) [41, 42], which involves refining an OF estimation from a previous iteration or from a coarser image scale, i.e., propagating a refined OF from a coarser to a finer image scale and iterating on each image scale. The 
two features mentioned above increase robustness to noise, brightness differences, and larger displacements, leading to improved accuracy of the OF calculation.

An extension of the 2D OF method of Farnebäck [40] to deal with volumetric data (Farneback-3D) has been recently implemented (https://pypi.org/project/farneback3d) and this 3D OF calculation method was used in TomoFlow that is presented here. For more information on the iterative multiscale (pyramidal) approach for 3D OF calculation used in TomoFlow, the reader is referred to Supplementary Method Description 1 section of Supplementary Material.

The concept of OF can be employed for 3D elastic and rigid-body matching, as explained hereafter. Let $V$ be a reference volume with a high SNR (e.g., a subtomogram average), and let $r$ be the $(x, y, z)$ coordinates of $V$. Moreover, let $S$ be an MW-corrected and rigid-body aligned subtomogram. Then, the following relationship between $V$ and $S$ is valid:

$$
S=V\left(r+\delta_{O}(r)+\delta_{C}(r)+\delta_{A}(r)\right)+N
$$

Where $\delta_{O}$ represents the voxel-to-voxel relationship between $V$ and $S$ to have an ideal rigid-body alignment, i.e., it stands for the rigid-body alignment imperfections of $S$; $\delta_{C}$ represents the relationship between the voxels of $V$ and $S$ to have an ideal elastic matching, i.e., it stands for the conformational variability of the subtomograms with respect to the reference; $\delta_{A}$ represents the residual anisotropies of the subtomogram after MW and Contrast Transfer Function (CTF) correction; $N$ is the subtomogram background noise.

3D dense OF between $V$ and $S$ can provide an estimate of the three voxel relationships combined, i.e., $\delta_{O}+\delta_{C}+\delta_{A}$, challenged by the noise $N$. Luckily, recent 3D dense OF implementations are loyal to the signal and can operate under very low SNR, especially when helped by a mask that eliminates the background. We can apply algorithms that can minimize the data anisotropies, i.e., $\delta_{A}$, mainly in terms of MW correction $[27,28]$ and 3D CTF correction $[43,44]$. Hence, 3D dense OF between $V$ and $S$ in the aforementioned conditions is an estimate of their rigid-body and elastic relationships combined:

$$
O F(V, S) \approx \delta_{O}(r)+\delta_{C}(r)
$$

When OF is calculated, it can be applied to the voxels of $V$ to estimate $S$; this operation is called warping, and the result $\hat{S}$ will be an estimate of $S$ with high SNR that we will refer to as a "matched" 
subtomogram. An illustration of OF calculation and its usage in matching subtomograms is shown in Figure 2.

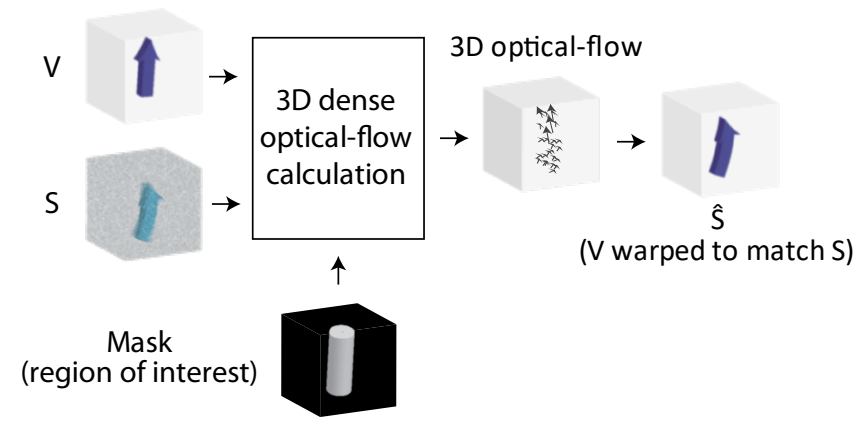

Figure 2 Illustration of the employment of 3D dense OF for elastic and rigid-body matching of subtomograms: $V$ is a volume with a high SNR (e.g., a subtomogram average). $S$ is a volume with a low SNR and contains a similar object as $\mathrm{V}$ but at a different conformation and a slightly different orientation and position (e.g., a MW-corrected subtomogram that was rigid-body aligned but not perfectly). $\hat{S}$ is an estimation of $S$ found by warping $V$ using 3D $\mathrm{OF}$, i.e., $\hat{S}$ is a matched version of $\mathrm{S}$ using $\mathrm{V}$ and the OF.

\section{2. $M W$ correction and refining the rigid-body alignment}

In conventional StA and classification, a compensation for the MW is commonly performed using a scoring function that operates in the Fourier space region excluding the MW [45]. However, for analyzing individual cryo-ET subtomograms in real space, the MW artifacts should be corrected.

In the previous subsection, we have shown that MW correction is needed to minimize the data anisotropies of an analyzed subtomogram, i.e., $\delta_{A}$ in equation (3). Also, we have shown that the 3D dense OF can match a subtomogram with a reference in terms of the rigid-body and elastic relationships combined, i.e., $\delta_{O}(r)+\delta_{C}(r)$ in equation (4). Therefore, to analyze the conformational variability of subtomograms, it is essential to correct the MW and disentangle between OF's rigid-body and elastic matching, which we will discuss in this subsection.

Several methods for MW correction were proposed in the literature $[25,26]$ and any of them could be used in conjunction with the proposed method. Here, we use a simple method to fill the MW of each subtomogram in Fourier space by the corresponding section from the aligned average, which was initially implemented in Eman2 [46]. We incorporate the MW correction in an iterative rigid-body refinement procedure based on OF subtomogram matching. The procedure is shown in Figure 3 has the following steps: 
Step 1. Rigid-body alignment: this can be achieved using StA methods [24, 46-49] to obtain a table of rigid-body parameters (angles and shifts) that can align the subtomograms to a global subtomogram average. Here, we use reference-free rigid-body alignment using the StA protocol in [49].

Step 2. MW correction of subtomograms: this can be done using any MW correction algorithm (e.g., LoTToR [28]). Here, we fill the subtomogram MW region in Fourier space by the corresponding region of an aligned subtomogram average, as initially implemented in Eman2 [46].

Step 3. Alignment of the MW-filled subtomograms with the average using the StA table: after filling the MW of the subtomograms, we apply the rigid-body alignment of the latest StA table and obtain MWcorrected and aligned subtomograms.

Step 4. Calculation of 3D OF between the subtomogram average and each MW-filled and aligned subtomogram: this should be done in the presence of a mask that determines the region of interest, which can be obtained by thresholding the subtomogram average and applying morphological operations such as dilating and closing. At this step, we also calculate warped versions of the subtomogram average using 3D OF calculated for each subtomogram. These warped versions of the subtomogram average are referred to as "matched subtomograms".

Step 5. Rigid-body alignment of "matched subtomograms" against the subtomogram average: this step disentangles the rigid-body and elastic matchings of OF by searching for rigid-body alignment of "matched subtomograms" against the subtomogram average. We perform this step using Fast Rotational Matching (FRM) [49].

Step 6. Updating the table of rigid-body alignment and calculating a new subtomogram average: this is done by combining the initial rigid-body alignment parameters (from the StA table) with the rigid-body refinement parameters obtained in the previous step, which is done by multiplying the corresponding rotational matrices and finding the rigid-body parameters for the resultant matrix [50].

This process can be repeated (1-3 times is usually enough), restarting at Step 2, which results in subtomograms that are MW-corrected and whose rigid-body alignment is refined. 


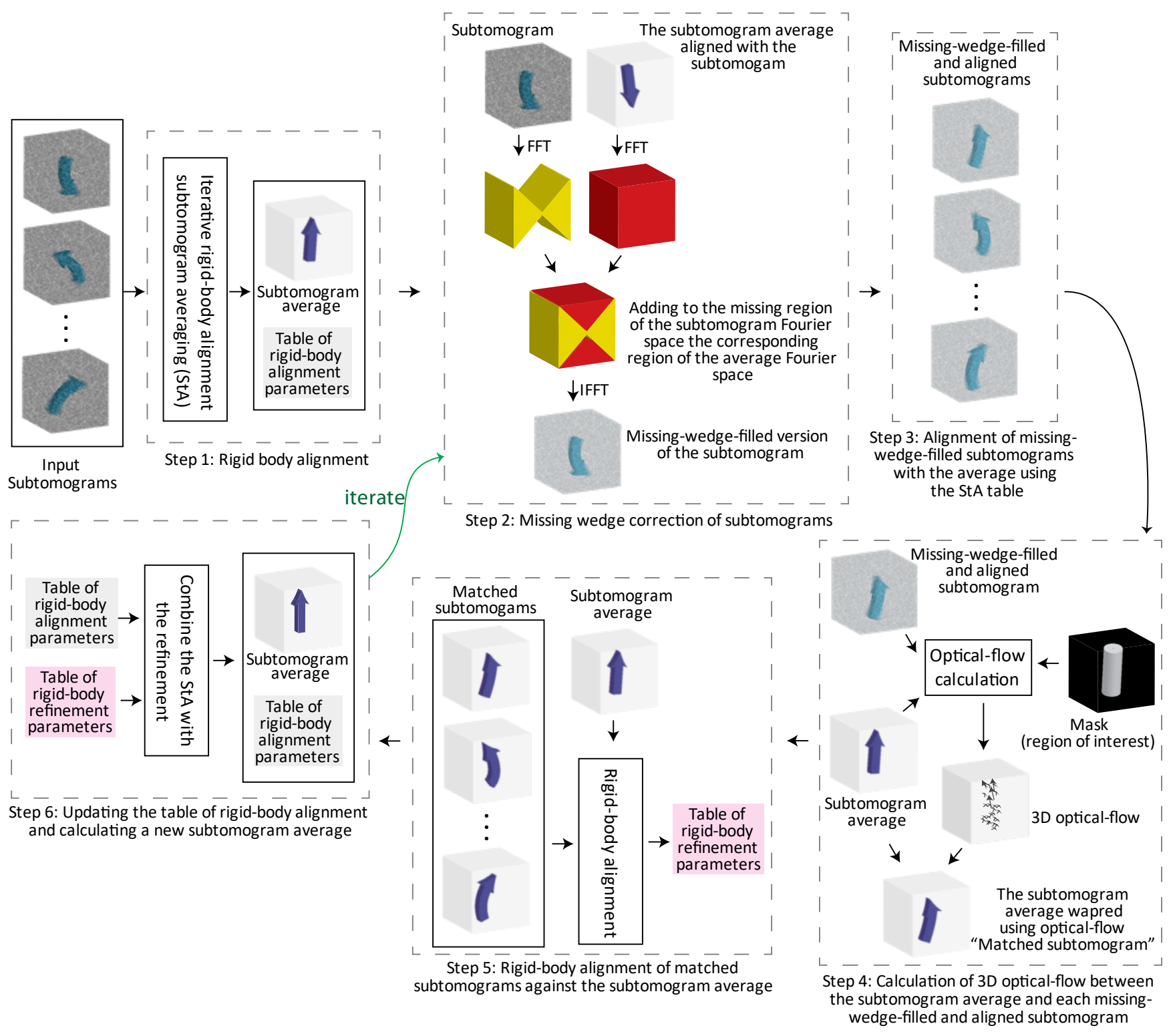

Figure 3 Pipeline for rigid-body alignment of subtomograms, MW correction, and refinement of the rigid-body alignment based on OF subtomograms matching.

\subsection{Analyzing the continuous conformational variability based on OF}

Assume that a set of subtomograms $\left\{S_{i}\right\}$ has undergone MW correction and rigid-body alignment and refinement, following the procedure presented in the previous subsection and Figure 3 . Then let $V$ be a reference for the target macromolecule contained in $\left\{S_{i}\right\}$, e.g., the corresponding subtomogram average of $\left\{S_{i}\right\}$. Concurrently, OF calculation in the presence of the mask of the region of interest, between $V$ and each subtomogram in $\left\{S_{i}\right\}$, will mainly stand for the term $\delta_{C}(r)$ in equation (4) since $\delta_{O}(r)$ was minimized as a result of the rigid-body refinement. In other words, the OF after MW 
correction and rigid-body alignment refinement represents the elastic matching relationship between the voxels of the $V$ and $S_{i}$.

Now let set $\left\{F_{i}\right\}$ be the set of OFs between $V$ and $\left\{S_{i}\right\}$ :

$$
F_{i}=O F\left(V, S_{i}\right)
$$

Let $S_{i} \in R^{l * m * n}$, then $F_{i} \in R^{3 * l * m * n}$ since OF gives a 3D vector for each voxel in $V$ to its matching voxel in $S_{i}$. We note here that cryo-ET subtomograms are usually cubic volumes, therefore $l=m=n$.

The corresponding Gram matrix $G$ of $\left\{F_{i}\right\}$ can be defined as:

$$
G_{i, j}=\operatorname{vec}\left(F_{i}\right)^{T} * \operatorname{vec}\left(F_{j}\right)
$$

Where $\operatorname{vec}($.$) is the vectorization operation, i.e., it reshapes the matrix to a single column.$

Once the Gram Matrix is found, a dimensionality reduction technique can be applied (e.g., PCA) to obtain an essential conformational space.

\subsubsection{Interactively processing the conformational space by selective 3D averages and animating trajectories}

In the conformational space, each point assigns an OF, which in turn assigns a subtomogram. Close points represent subtomograms of similar conformations and vice versa. Dense regions in the conformational space can be grouped interactively, and the corresponding subtomograms can be averaged. Comparing the subtomogram averages from different groups can help understand the conformational changes of the complex in the given set of subtomograms.

Data distribution paths (trajectories) can be interactively determined in the conformational space, by choosing a set of points $\left\{P_{i}\right\}$ across the data distribution. The motion of the macromolecule can be obtained by displacing the reference (e.g., the subtomogram average) along the trajectory determined by points $\left\{P_{i}\right\}$, as explained below.

Once a trajectory is determined in the conformational space (points $\left\{P_{i}\right\}$ are chosen), the inverse mapping should be applied (e.g., using inverse PCA) on $\left\{P_{i}\right\}$, and the result will be a set of vectors $\left\{\widehat{G}_{i}\right\}$ of the same length as the columns of the Gram matrix $G$ given in equation (6). To proceed on how 
animations can be obtained, we need to rewrite equation (6) alternatively. Let $O$ be the matrix of vectorized OFs in its columns as follows:

$$
O_{i}=\operatorname{vec}\left(F_{i}\right)
$$

Then, $G$ can be written as:

$$
G=O^{T} * O
$$

Hence, any column of $G$ can be expressed as:

$$
G_{i}=O^{T} * \operatorname{vec}\left(F_{i}\right)
$$

We take advantage of the representation of $G$ in equation (9) to approximate a set of OFs $\left\{\widehat{F}_{l}\right\}$ that correspond to $\left\{\widehat{G}_{i}\right\}$ as follows:

$$
\operatorname{vec}\left(\widehat{F}_{l}\right) \sim\left(O^{T}\right)^{+} * \widehat{G}_{i}
$$

Where the $(.)^{+}$is the Moore-Penrose matrix pseudoinverse operation

The retrieved set of $\left\{\operatorname{vec}\left(\widehat{F}_{l}\right)\right\}$ can be reshaped to OFs:

$$
\widehat{F}_{l}=\operatorname{vec}_{3 * l * m * n}^{-1}\left(\operatorname{vec}\left(\widehat{F}_{l}\right)\right)
$$

The set of retrieved OFs, i.e., $\left\{\widehat{F}_{l}\right\}$, can be used to warp the input reference, which will generate a set of Trajectory Volumes $\left\{\widehat{T V}_{l}\right\}$ that represent the set of trajectory points $\left\{P_{i}\right\}$. Finally, displaying $\left\{\widehat{T V}_{l}\right\}$ shows a movie-like animation of the reference while traversing the selected trajectory.

\section{Results}

This section first provides a step-by-step showcase and evaluation for the proposed method, TomoFlow, on simulated datasets. Then, it shows an application of TomoFlow on an experimentally obtained dataset for nucleosomes in situ.

\subsection{Tests on simulated datasets with continuous and discrete conformational variability}

The experiments presented in this section were carefully designed to demonstrate the ability of the method to retrieve continuous and discrete conformational variabilities under simulated microscope 
conditions. These experiments are not claimed realistic in terms of their biological significance; rather, they are as realistic as possible in terms of the sophistication of simulating noise, MW artifacts, CTF, and radiation damage compared to other works $[22,23,51]$. For a quantitative assessment of the algorithm for mapping conformations while disentangling it from the subtomographic-approach limitations such as MW and rigid-body (angular and shift) variability, the reader is referred to Supplementary Experiment 1 section of Supplementary Material.

\subsubsection{Simulating datasets with discrete and continuous macromolecular conformational variability}

In order to test the proposed method, we synthesized two conformationally different datasets, each with different noise intensities.

The first dataset simulates discrete conformational variability. It was created using Normal Mode Analysis (NMA) [52]. We will call this dataset the "NMA-dataset". NMA is a method for molecular mechanics simulation with wide usage in flexible fitting and conformational variability analysis [6, 13, 18, 53]. This dataset comprises 999 subtomograms at three simulated conformations of chain $A$ of the atomic PDB:4AKE structure of adenylate kinase. More precisely, we synthesized 333 subtomograms for each of the three conformations simulated using normal modes 7 and 8 of chain $A$ of the atomic PDB:4AKE structure (in this example, we have chosen to simulate the data using the first two non-rigidbody normal modes of the atomic structure, considering that the first six modes correspond to rigidbody movements and are commonly not used). We performed NMA using the ContinuousFlex plugin with default parameters [13]. The three conformations in this dataset correspond to the following normal mode amplitudes (mode 7 , mode 8$) \in\{(-100,0),(100,0),(0,100)\}$. The ground-truth conformational space for NMA-dataset is determined by the amplitudes along normal modes 7 and 8 . A visual representation of this space and the conformations it contains is presented in Figure 4 (A).

The second dataset simulates continuous conformational variability. It was created using Molecular Dynamics (MD) [54]. We will call this dataset the "MD-dataset". MD is a simulation approach for exploring conformational dynamics by generating trajectories describing a structure evolving over time. This dataset comprises 1000 subtomograms representing a continuum of conformations generated using an MD trajectory between two conformations of adenylate kinase chain A from the PDB structures 
PDB:4AKE (most open conformation) and PDB:1AKE (most closed conformation). We simulated this trajectory using GENESIS [55]. The ground-truth conformational space of the MD-dataset will be presented here by its first two principal axes (PCA). A visual representation of this space and the conformations it contains is presented in Figure 4 (B).
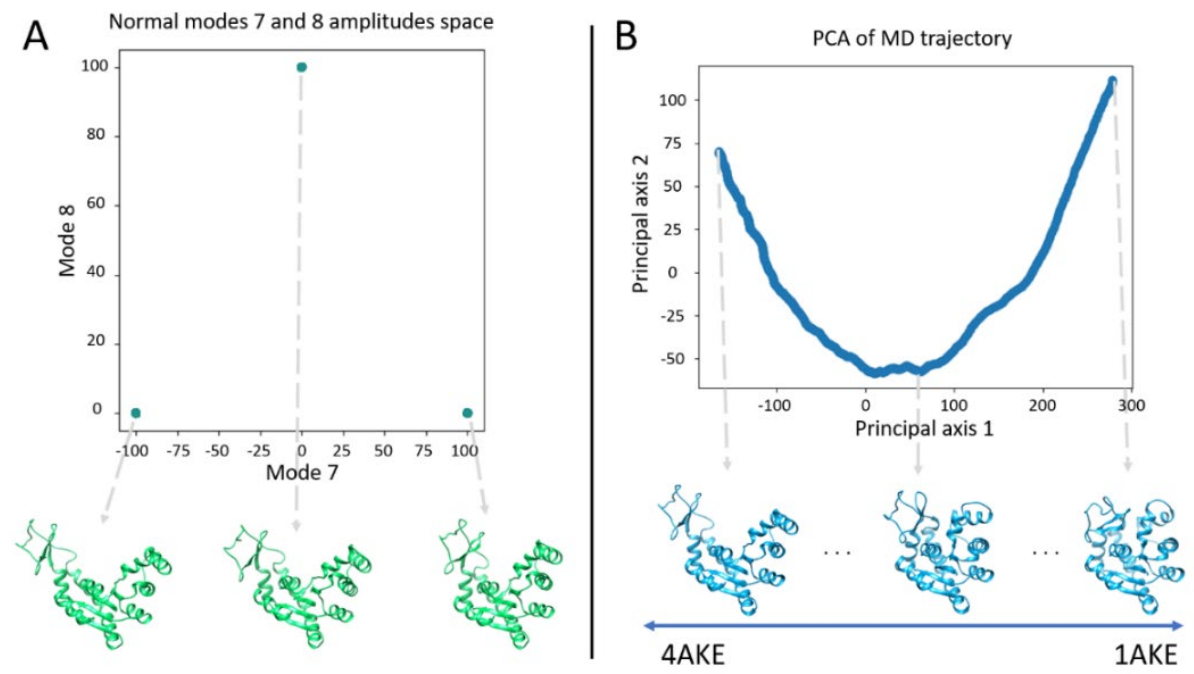

Figure 4 Ground-truth conformational spaces for the two simulated datasets. (A) NMA-dataset: mode 7 and 8 amplitude space with the corresponding three conformations that it contains. (B) MD-dataset: principal axes 1 and 2 showing a continuum of conformations (MD trajectory) between the PDB structures 4AKE (most open conformation) and 1AKE (most closed conformation).

While simulating data, we followed the best practices presented in the literature $[18,22,23,51]$ to make the data challenging while keeping the objectives clear and letting the method achieve its goals. For each subtomogram, we convert the PDB structure that represents the desired conformation (i.e., either one of the three conformations in the NMA-dataset or one of the continuum of conformations in the MD-dataset) to a volume of size $64^{3}$ voxels and voxel size of $2.2 \AA^{3}$ [56]. Then, we lowpass filter the volume to $6 \AA$ resolution in order to simulate radiation damage and other effects such as data misalignments incorporated at the tomogram reconstruction step (skipping lowpass filtering would result in better retrieval of conformational variability but is less realistic). Afterward, we rotate and shift this volume in 3D space using random Euler angles and random $x, y, z$ shifts in the radius of 5 voxels from the center. To obtain a tilt series, we project the rotated and shifted volume using tilt values $-60^{\circ}$ to $+60^{\circ}$ with $2^{\circ}$ step. We simulate microscope conditions by adding noise and modulating the tilt series with a CTF of defocus $-1 \mu \mathrm{m}$. Then we add noise again (half of the noise will be modulated by the CTF, and the other half will not). The same procedure is repeated for three different SNR values $(0.1,0.03$, 
0.01) and without noise. Then, we invert the CTF phase. Finally, we reconstruct a volume (our synthetic subtomogram) using a Fourier reconstruction method [57]. Figure 5 shows four examples of the simulated subtomograms (without noise and at the three different SNR values) for the same conformation, orientation, and position of the macromolecule, along with the corresponding ideal (ground-truth) density volume (the volume with no noise and no missing wedge artifacts, which is not a result of the reconstruction but obtained by converting the atomic structure of that conformation). To give the reader an idea of the resolutions of these subtomograms, we compared them with the groundtruth volume of the same conformation and found the resolutions of $6.4 \AA, 13.9 \AA, 19.9 \AA$ and $23.6 \AA$ for the simulated subtomogram without noise and with SNR of 0.1, 0.03, and 0.01, respectively (volumes in Figure 5), based on the Fourier Shell Correlation (FSC) between the non-masked volumes and the FSC threshold of 0.5 .

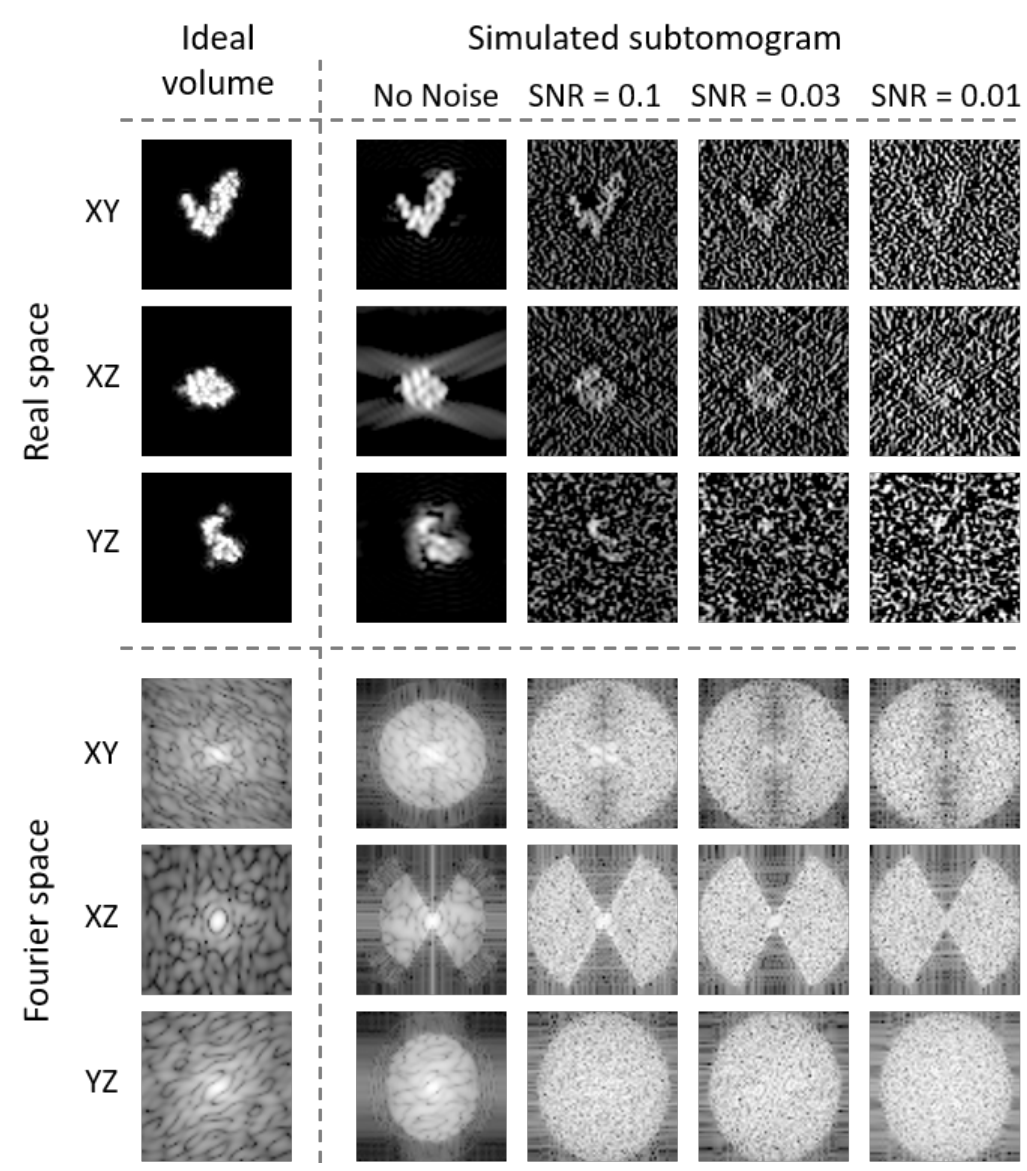

Figure 5 Central slices in real and Fourier spaces of a simulated subtomogram without noise and at different SNRs compared to the corresponding ideal volume. 


\subsubsection{Rigid-body alignment and refinement with MW correction}

We applied the proposed procedure in 2.2 and Figure 3 for rigid-body alignment and refinement with MW correction on the simulated datasets as follows:

Step 1. Performed reference-free rigid-body alignment using the StA protocol in [49]. This StA protocol uses an exhaustive angular search (with FRM), a shifting search within a region of interest, and MW compensation. The shifting search was set to the range of 10 voxels from the center, and the maximum searched normalized frequency to 0.25 . The iterative alignment was performed for 15 iterations (complete stability was achieved in the rigid-body alignment parameters and the resulting average).

Step 2. Filled the MW region in Fourier space for each subtomogram by the corresponding region of the aligned subtomogram average.

Step 3. Rigid-body aligned the MW-corrected subtomograms.

Step 4. Calculated the 3D OFs between the subtomogram average and each MW-filled and aligned subtomogram using Farneback-3D after multiplying both volumes with a mask. We generated this mask by binarizing the subtomogram average, dilating it by a structural element of size three, keeping its largest connected component, and smoothing its boundaries with a Gaussian filter of standard deviation equal to two. This step results in "matched subtomograms". In all experiments in this article, Farneback3D was run with a 2-level volume pyramid of scaling factor of 0.5 (meaning a pyramid with the levels of $64^{3}$ and $32^{3}$ voxels in the case of these two test datasets), a window size of $10 \times 10 \times 10$ voxels for integrating the displacement field over a neighborhood of each voxel, 10 iterations of the algorithm at each pyramid level, and with default values of all other parameters. It should be noted that $32^{3}$ voxels is the coarsest pyramid level allowed by Farneback-3D.

Step 5. Performed rigid-body alignment of "matched subtomograms" against the subtomogram average using FRM (the same method used for StA but here without MW compensation) in the range of 4 voxels from the center.

Step 6. Combined the StA table with the rigid-body refinement parameters obtained in the previous step and calculated a new subtomogram average (refined reference). 
We iterated the MW correction and rigid-body refinement process by first performing Step 1 once and Steps 2-6 three times. Table 1 shows the obtained rigid-body alignment results before and after applying this refinement algorithm. They show that the refinement globally reduces the distances between the estimated and ground-truth rigid-body parameters (angles and shifts) in the presence of noise and conformational variability (Table 1).

\subsubsection{Conformational variability analysis}

The datasets are ready for continuous conformational variability analysis after applying the MW correction and rigid-body refinement algorithm. Subsequently, we calculated the OFs between the refined reference and the $\mathrm{MW}$ corrected and rigid-body aligned subtomograms for each dataset. Then, we found the Gram matrix of OFs based on equations (5-6) and applied PCA. The conformational space, represented by the space of the first two principal vectors, for each dataset, at different noise intensities, is shown in Figure 6. A comparison between the ground-truth conformational spaces in Figure 4 and the retrieved conformational spaces in Figure 6 shows that i) for the NMA-dataset, the separation between the three conformations in the retrieved conformational space is evident for all the tested noise intensities, and ii) for the MD-dataset, the trajectory is more evident for lower noise intensity (higher SNR), and it is the least evident when the SNR is 0.01 .

\begin{tabular}{|c|c|c|c|c|c|c|}
\hline \multirow{2}{*}{ Dataset } & \multirow{2}{*}{ Noise } & \multirow{2}{*}{$\begin{array}{l}\text { Before/After } \\
\text { Refinement }\end{array}$} & \multicolumn{2}{|c|}{ Angular distance [deg] } & \multicolumn{2}{|c|}{ Shifting distance [vox] } \\
\hline & & & Mean & STD & Mean & STD \\
\hline \multirow{8}{*}{ NMA-dataset } & \multirow{2}{*}{ No Noise } & Before & 2.8 & 1.5 & 1.9 & 0.2 \\
\hline & & After & 2.5 & 1.4 & 0.9 & 0.1 \\
\hline & \multirow{2}{*}{$S N R=0.1$} & Before & 2.8 & 1.5 & 1.2 & 0.2 \\
\hline & & After & 2.5 & 1.3 & 1.2 & 0.2 \\
\hline & \multirow{2}{*}{ SNR $=0.03$} & Before & 3.1 & 3.1 & 1.3 & 0.4 \\
\hline & & After & 2.4 & 2.3 & 1.2 & 0.4 \\
\hline & \multirow{2}{*}{ SNR $=0.01$} & Before & 17.0 & 39.3 & 2.7 & 2.5 \\
\hline & & After & 16.5 & 39.7 & 2.6 & 2.4 \\
\hline \multirow{8}{*}{ MD-dataset } & \multirow{2}{*}{ No Noise } & Before & 3.3 & 2.5 & 1.4 & 0.2 \\
\hline & & After & 3.1 & 2.4 & 1.0 & 0.2 \\
\hline & \multirow{2}{*}{ SNR $=0.1$} & Before & 2.6 & 1.6 & 1.5 & 0.2 \\
\hline & & After & 2.5 & 1.7 & 1.5 & 0.2 \\
\hline & \multirow{2}{*}{ SNR $=0.03$} & Before & 4.0 & 2.9 & 1.6 & 0.2 \\
\hline & & After & 3.9 & 2.7 & 1.4 & 0.2 \\
\hline & \multirow{2}{*}{$S N R=0.01$} & Before & 4.8 & 3.4 & 1.2 & 0.4 \\
\hline & & After & 4.5 & 3.1 & 1.0 & 0.3 \\
\hline
\end{tabular}

Table 1 Mean and standard deviation (STD) of the absolute distance between ground-truth and estimated rigidbody parameters via StA before and after the proposed rigid-body refinement algorithm applied to NMA-dataset and MD-dataset. 
To give the reader a sense of what the method can achieve when applied to challenging datasets expected in experimental studies, we will base our evaluation of the retrieved conformations for both datasets on the most challenging noise case ( $S N R=0.01)$, in which the molecule is barely visible in the subtomograms (Figure 5).

\subsubsection{Conformational variability analysis for the NMA-dataset}

This subsection presents the results of analyzing the conformational variability in the NMA-dataset at SNR $=0.01$ using TomoFlow. Figure 7 presents the retrieved conformational space of this dataset, highlighting three distinct groups of points in this space and their corresponding subtomogram averages. Moreover, each group average is compared with its ground-truth atomic structure at the corresponding conformation by docking this atomic structure into the average volume and displaying the volume at $40 \%$ opacity.

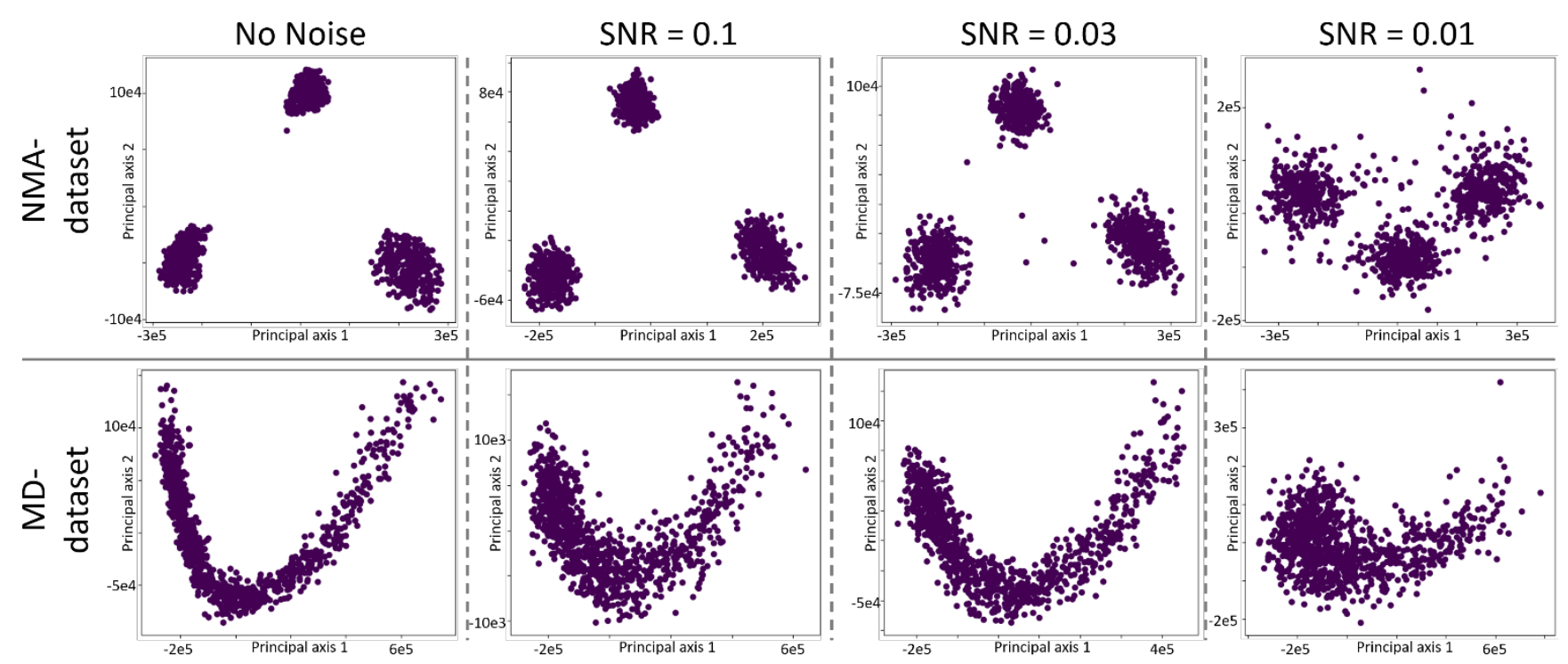

Figure 6 Plots showing the output conformational spaces found by TomoFlow on NMA-dataset and MD-dataset for different noise intensities. The ground-truth conformational spaces for these datasets are shown in Figure 4. We note here that only the distribution should be compared with the ground-truth, which indicates that the interrelationship between the conformations was retrieved correctly (i.e., similar conformations were mapped to close points and vice versa); the limits of the horizontal and vertical axes do not correspond to those of the groundtruth since the ground-truth conformational space relates atomic structures (NM amplitudes or PCA of MD trajectory) and retrieved conformational space relates OFs. 
We compared the three obtained subtomogram averages with the ground-truth volumes of the corresponding conformations (the atomic structures of these conformations converted into volumes), based on the FSC between non-masked volumes and the FSC threshold of 0.5. The obtained resolutions

of the three volumes from left to right in Figure 7 are $9.6 \AA$, $9.5 \AA$, and $9.5 \AA$, respectively. Note here that each of the three volumes was obtained by averaging around 300 subtomograms (Figure 7) and recall that the resolution of an individual subtomogram is around $24 \AA$ for SNR $=0.01$. Thus, we observe that the resolution was improved by more than $50 \%$ by averaging only 300 subtomograms, which were aligned in terms of molecular conformation, orientation and position using TomoFlow.

Since the conformational variability in this dataset is discrete, animations are not presented for this dataset.

\subsubsection{Conformational variability analysis for the MD-dataset}

This subsection presents the results of analyzing the conformational variability in the MD-dataset at $\mathrm{SNR}=0.01$. Figure $8(\mathrm{~A})$ presents the retrieved conformational space of this dataset, highlighting six selected groups of subtomograms in this space and their corresponding averages. Moreover, each group average is compared with the ground-truth atomic structure found as the group's centroid at the corresponding conformation by docking this atomic structure in the average volume and displaying the volume at $40 \%$ opacity (Figure $8(\mathrm{~A})$ ). Regarding the number of groups and their locations, it is encouraged to try more and fewer groups, which may help to better understand the conformational variability. TomoFlow software provides a graphical interface for an interactive selection of the regions in the low-dimensional conformational space in which subtomograms will be summed and their averages computed. Usually, the averages will be calculated from the densest regions (the regions with the largest numbers of points, i.e., subtomograms). The density of points can be visualized using different shades of coloring the points (from the lowest density indicated by the lightest color to the highest density indicated by the darkest color). The size (radius) of each subtomogram averaging region in the conformational space should be selected carefully. Indeed, small-radius regions should still contain enough subtomograms to produce subtomogram averages with sufficiently attenuated noise and MWinduced deformations. Also, large-radius regions should not result in smooth subtomogram averages because the conformational differences between such smooth averages from different regions may not 
be distinguishable. In general, the higher the resolution and the number of subtomograms, the more it is possible to select denser regions of smaller radii and reveal the conformational variability of the targeted macromolecule, and vice versa.

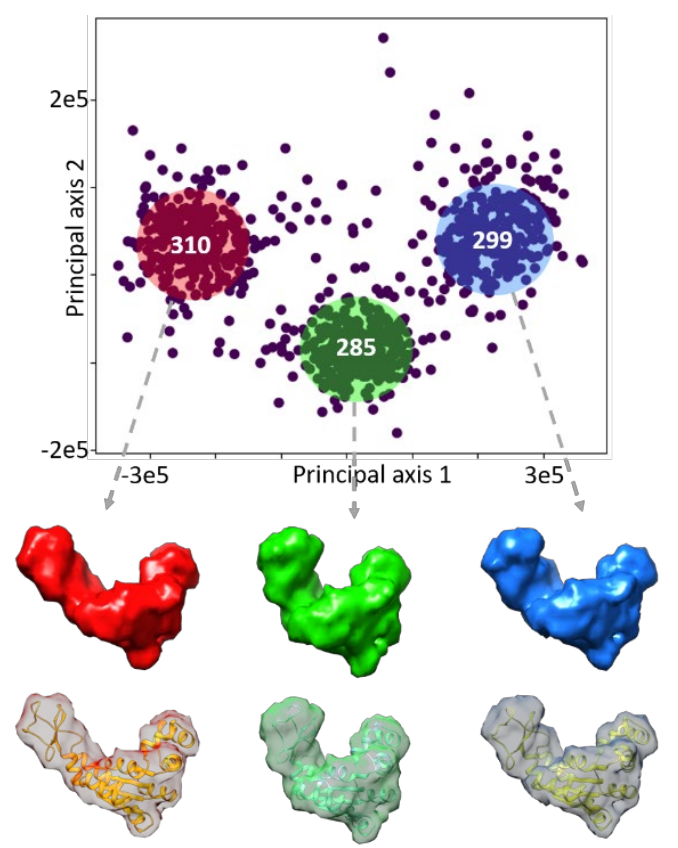

Figure 7 The conformational space found using TomoFlow on the NMA-dataset with SNR $=0.01$ (the ground-truth conformational space is shown in Figure 4). The shown volumes are averages of three groups of subtomograms identified by the highlighted ellipses. The number shown inside an ellipse corresponds to the number of points it encloses. The bottom row displays the averages at $40 \%$ opacity with their corresponding ground-truth atomic structure docked inside for comparison.

The averages obtained from the six selected groups of subtomograms in Figure 8 (A) (the selected regions of the conformational space of the MD-dataset at SNR $=0.01$ ) were compared with the corresponding ground-truth volumes (the volumes obtained by converting the atomic structure of the group's centroid at the corresponding conformation), based on the FSC between non-masked volumes and the FSC threshold of 0.5 . The obtained resolutions of the six volumes from left to right in Figure 8 are $9.8 \AA, 9.7 \AA$, $9.7 \AA$, $9.8 \AA, 11.6 \AA$, and $15.8 \AA$, respectively. It can be noted that these resolutions are correlated with the numbers of subtomograms averaged in each group (the numbers shown in Figure 8 (A)). For instance, the subtomogram average of the lowest resolution (15.8 $\AA$ ) was obtained from the lowest number of subtomograms (80). Also, note that the resolution is $11.6 \AA$ for averaging 101 subtomograms and it is below $10 \AA$ for averaging 112 subtomograms (the averaging of 112-185 
subtomograms resulted in the resolution of 9.7-9.8 $\AA$ ). Thus, we observe that the resolution can improve by more than $50 \%$ with respect to the resolution of an individual subtomogram ( $24 \AA$ for SNR $=0.01$ ) by averaging as little as around 100 subtomograms, if these subtomograms are aligned in terms of molecular conformation, orientation and position using TomoFlow.

Figure 8 (B) presents an animation following the data distribution manifold. This animation is generated by applying the inverse PCA mapping on the identified points (the ten numbered red points shown in the space), then using equations (10-11) to generate the corresponding OF for each point. These generated OFs are then used to warp the subtomogram average (the global average found after refinement) to generate volumes. The latter volumes correspond to the animation frames, shown as the numbered volumes at the bottom row. When these volumes are displayed sequentially, they show an animation that reveals the MD trajectory used to create the data. This animation is provided in the supplementary material (Supplementary Movie 1).
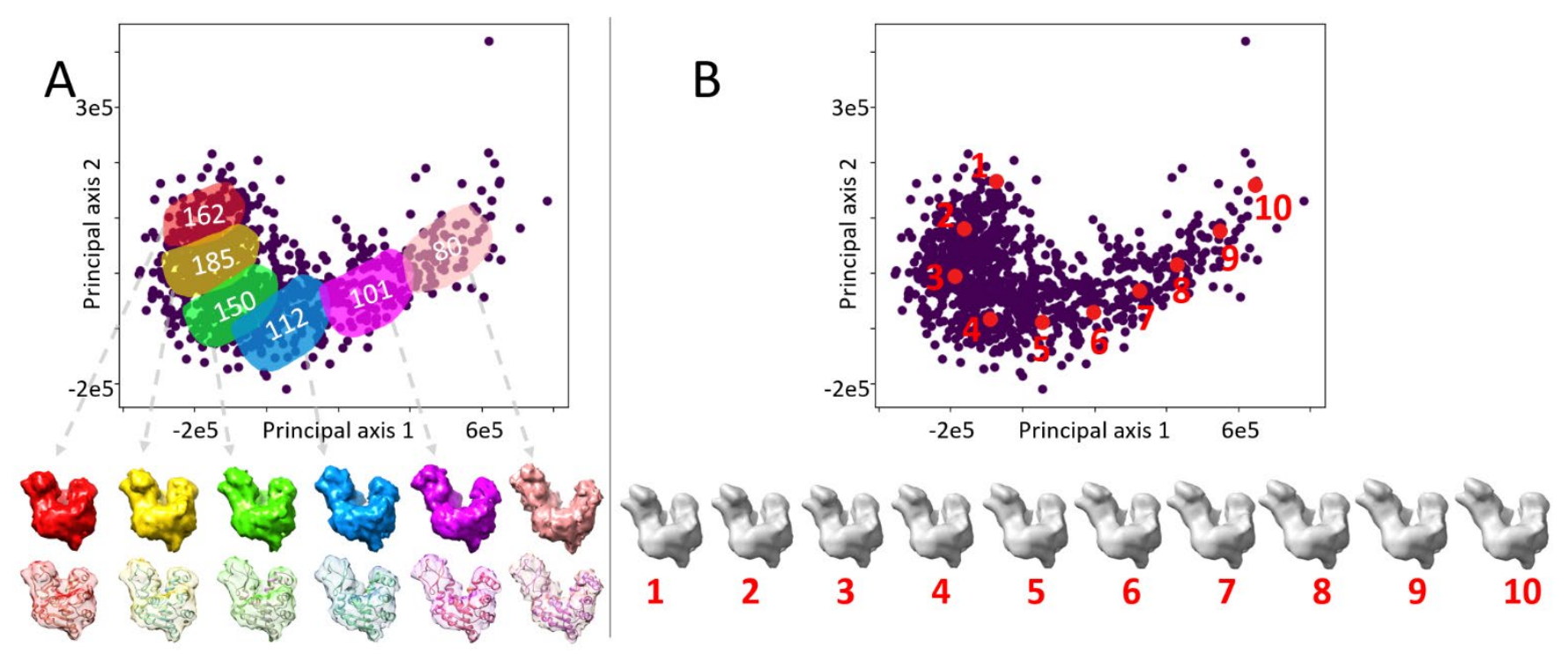

Figure 8 Continuous conformational variability analysis via selective subtomogram averages and animation using TomoFlow conformational space of the MD-dataset with SNR $=0.01$. (A) Subtomogram averages of six groups of subtomograms identified by the highlighted areas of the conformational space. The number shown inside a highlighted area corresponds to the number of points it encloses. The bottom row displays the averages at $40 \%$ opacity with their corresponding ground-truth atomic structure (group centroid) docked inside for comparison. (B) Displacement of the global subtomogram average along the direction of the data distribution in the conformational space (molecular motion along a trajectory); animation consisting of ten frames represented by a sequence of red dots (from 1 to 10, see also Supplementary Movie 1). The ground-truth conformational space is shown in Figure 4. 


\subsection{Conformational variability of nucleosomes in situ}

This section describes the application and results of TomoFlow on nucleosomes in situ, in their interphase nucleus context. We use a dataset containing 666 subtomograms (EMPIAR-10679) of nucleosomes extracted from cryo tomographic reconstruction of a vitreous section of a high-pressure frozen Drosophila embryonic brain $[17,18]$, with a subtomogram volume size of $64^{3}$ and voxel size of 4.4 $\AA^{3}$. Reference-free rigid-body StA of this dataset was previously performed using SubTomogramAveraging script of Artiatomi (https://github.com/uermel/Artiatomi) [58] and the average was deposited in EMDB under the accession code EMDB-12699 [18]. For the full details about this dataset acquisition and StA, we direct the reader to Section S1 (supplementary materials) of [18].

The nucleosome conformational variability detected in this dataset in previous works $[17,18]$ was mainly described as gapping and breathing motions of the nucleosome [35]. However, these conformational variabilities were previously identified either via manual measurements [17] or via selecting a set of motions of interest (set of normal modes) for data analysis [18]. Here, our goal is to check if TomoFlow can detect the expected movements without using any prior knowledge about the nucleosome conformational variability.

First, we used the StA parameters to reproduce the global subtomogram average. We used the average to generate a mask of the region of interest, which was then used to refine rigid-body alignment and analyze the conformations in the data. We generated the mask by binarizing the subtomogram average, dilating it by a structuring element of size three, keeping its largest connected component, and smoothing its boundaries with a Gaussian filter of standard deviation equal to two. Second, we performed seven MW correction and rigid-body refinement iterations following the procedure shown in Figure 3 (the theoretical details of the MW correction and rigid-body refinement are explained in 2.2, and its practical implementation in 3.1.2). Third, we followed the procedure described in 2.3 to analyze the conformational variability after rigid-body alignment and MW correction of subtomograms. We applied PCA on the Gram matrix of OFs, and the conformational space determined by the first two principal axes is shown in Figure 9.

By inspecting the conformational space, we notice that the first principal axis has significantly larger variability than the second principal axis. We analyzed the variability carried along the first principal axis 
by analyzing two subtomogram averages and an animation generated along this principal axis. We selectively generated subtomogram averages from groups of points at the beginning and the end of the data distribution. The regions for the groups of points are shown as highlighted areas in Figure 9 (A), along with their corresponding subtomogram averages. The averages are generated for the MWcorrected and rigid-body aligned subtomograms. Also, we generated animation for the variability along the first principal axis, within the limits of the data distribution manifold represented by the line $D$ in Figure 9 (B). This animation is generated by estimating the OFs for ten points along line $D$, then warping the global subtomogram average using these estimated OFs. The resulting volumes are displayed sequentially to generate the animation (Supplementary Movie 2 and Figure 9 (B)).
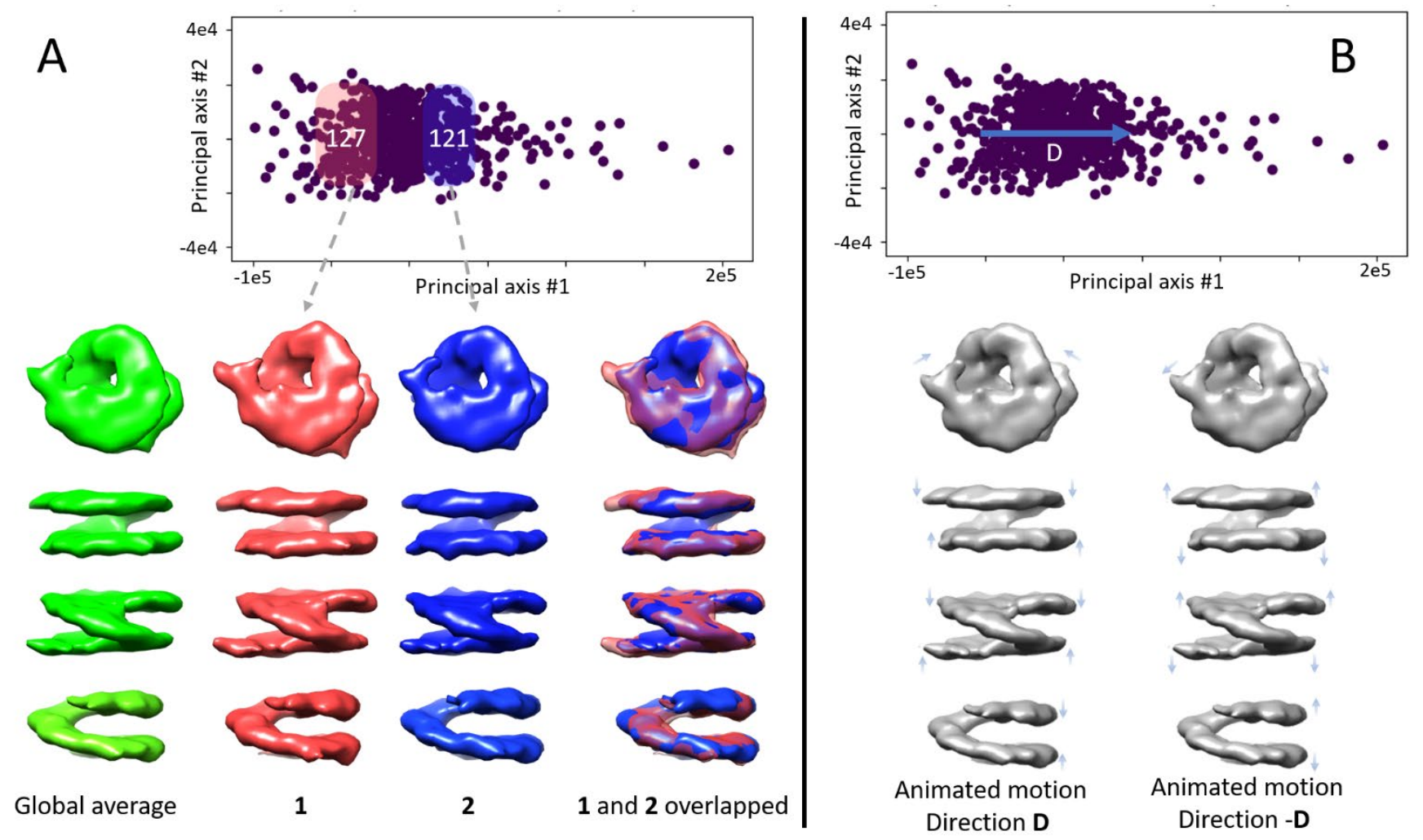

Figure 9 TomoFlow applied to cryo-ET dataset of nucleosomes in situ. (A) group averages for two regions specified by highlighted areas in the conformational space and the corresponding averages. The number inside a highlighted area indicates the number of subtomograms the area encloses. (B) an illustration showing the displacement of the global subtomogram average along the first axis in the limits of the data distribution shown by line $D$ in the conformational space. The arrows on the different views of the global average show the direction of the movement in this animation. The animation is provided in the supplementary material (Supplementary Movie 2). 
The main differences between the two group averages (Figure $9(A)$ ) and the motion observed along line D (Figure 9 (B) and Supplementary Movie 2) indicate that TomoFlow detected a combined breathing and gapping motion of the nucleosome, with the breathing motion more expressed. These results are consistent with those of our previous study of conformational variability using the same dataset [18] but without imposing any prior knowledge on the analysis of the conformational variability.

\section{Software implementation}

The software of TomoFlow is open-source and available as a part of the ContinuousFlex plugin of Scipion $[59,60]$. Our software provides a user-friendly Graphical User Interface (GUI). Using the default parameters, the current implementation takes 32 seconds to calculate an OF between a subtomogram and a reference of size $64^{3}$ voxels (tested on Nvidia Quadro RTX 5000). One calculation of OF per subtomogram is required per rigid-body refinement iteration, and one more OF calculation is required per subtomogram for continuous variability analysis. The OF calculation requires the most data processing time, and therefore, the OF calculation is GPU parallelized. The number of OFs that can be calculated simultaneously depends on the number of available GPUs (one OF calculation uses one entire GPU). Our method's second most time-demanding task is rigid-body alignment (used during rigid-body refinement), which we implemented using FRM [49]. We parallelized the FRM implementation using a Message Passing Interface (MPI) scheme. The number of subtomograms that can be processed simultaneously using our parallelized version of FRM depends on the number of available CPU threads (one subtomogram is processed using one CPU thread). Three refinement iterations of a dataset of 1000 subtomograms of size $64^{3}$ require around 36 hours using a single GPU card and 20 CPU threads (tested on Nvidia Quadro RTX 5000 and 2.2 GHz Intel Xeon Silver 4214 CPU processors). TomoFlow requires StA to be performed in advance. TomoFlow software provides one StA implementation that is based on FRM [49], but it also allows importing StA parameters obtained with Dynamo or TOM2-toolbox (other StA software may be made compatible with TomoFlow in the future).

\section{Discussion and conclusion}

Cellular cryogenic electron tomography (cryo-ET) is currently undergoing its "resolution revolution" reaching a near atomic resolution in situ [61] and allowing studying macromolecules in their 
physiological environment that affects their conformational landscape $[18,62]$. This article presented TomoFlow method that addresses continuous macromolecular conformational variability captured in cryo-ET subtomograms. TomoFlow employs dense 3D optical-flow, posterior to conventional StA, to refine the rigid-body alignment and analyze the conformational variability. The method maps the subtomograms to a space of conformations and allows i) interactively generating subtomogram averages of different conformations (without classification), and ii) navigating the conformational space of the macromolecule via animations based on a reference (e.g., the global subtomogram average).

It should be noted that grouping similar structures and computing their averages for improving SNR, as it is done in the traditional StA workflows, is not the main objective of TomoFlow. Its main objective is to obtain the conformational landscape that can be easily visualized (in two or three dimensions determined by the first two or three principal axes) and explored in terms of molecular flexibility animations along different directions (animated displacements of a reference conformation). Yet, TomoFlow allows making such groups of similar structures and computing their averages, but in contrast to the traditional, discrete classification methods, the number of groups in TomoFlow is not defined prior to the analysis and it is selected according to the conformational distribution in the low-dimensional conformational space. Furthermore, using traditional StA workflows, less dominant conformations are likely to be undiscovered as being wiped out through the global or class averages blindly (no possibility of visualizing all conformations in a common frame and selecting the conformations to average accordingly). On the contrary, TomoFlow provides a visualization of the full conformational space and, thus, allows discovering less dominant conformations (as less dense regions in this space) and prevents from wiping such conformations out thanks to an interactive selection of the regions from which the averages will be calculated. However, TomoFlow is not designed for analyzing all types of structural variabilities such as macromolecular disassembly or binding and unbinding of ligands, but it can be combined with discrete classification methods to disentangle such structural variabilities and then analyze continuous intraclass variability using class averages as references instead of the global subtomogram average.

We presented the method with simplified yet sufficient mathematical derivations that are necessary for understanding and implementing it. We tested the method by synthesizing two datasets under challenging conditions and testing its capability in retrieving their ground-truth conformational spaces. 
The results of the tests with simulated data indicate that i) the proposed rigid-body refinement can improve the alignment quality in the presence of conformational variability, and ii) the proposed conformational variability analysis can accurately recover hidden conformations. Additionally, we tested TomoFlow using a cryo-ET dataset of nucleosomes in situ, which provided promising results coherent with previous findings using the same dataset [18] but without imposing any prior knowledge on the analysis of the conformational variability.

It should be reminded that better statistics (including those produced by PCA) are obtained for larger datasets. The cryo-ET datasets that can be collected (thus, analyzed by TomoFlow method) nowadays are still much smaller than those produced by single-particle cryo-EM. However, the goal of the PCA in Tomoflow is to reveal the major motions of the complex. In this context, a dataset of 2000 subtomograms that can be obtained nowadays may be considered large enough for such PCA and should allow revealing the main motions of the complex. In this article, we have shown the results of an experiment with 666 in-situ cryo-ET nucleosome subtomograms, which appears to be sufficient for revealing the breathing and gapping motions of the nucleosome, the two main motions of the nucleosome that have also been detected using two different methods in situ $[17,18]$ as well as in a theoretical study [35].

TomoFlow performs the analysis in real space. Hence, it requires MW correction since the MW compensation as in reciprocal space analysis is not possible in real space. MW correction algorithms exist and TomoFlow can work in conjunction with any of them. Here, we used a method for MW correction based on filling the MW region of subtomograms with the corresponding region of the global subtomogram average. A more advanced MW correction method can be used in the future and might lead to better results.

OF is a powerful and robust image analysis algorithm. Earlier OF approaches were detecting small changes, typically in a few pixels/voxels range. Recent OF methods, such as Farneback-3D used in TomoFlow, cope with this limitation by combining OFs from multiple scales (pyramid-scheme processing). Nevertheless, TomoFlow will be more efficient for smaller conformational variability in the data (for a systematic test of TomoFlow matching different conformational variability magnitudes, the reader can see Supplementary Experiment 1 in Supplementary Material). Additionally, Farneback-3D 
method enforces the smoothness of the motion field between the two given volumes (the volumes between which the OF should be calculated), which allows a correct calculation of the OF under very heavy noise and resisting against MW artifacts. However, the OF smoothness enforcement induces smoothness of the generated animation (a smooth version of the warped reference in each frame, e.g., Figure 8 (B)). Finally, obtaining animations requires Moore-Penrose pseudoinverse to be found for a large matrix given in equation (10). This matrix is defined as the matrix of column-wise vectorized OFs that can be three times the dimensions of the input subtomogram dataset; for instance, generating animations after processing a dataset that comprises 1000 subtomograms of volume size $64^{3}$ will require the inversion of $3^{*} 64^{3}$ rows by 1000 columns, which becomes computationally challenging for large datasets, mainly in terms of the required memory. However, a downsampling (e.g., by 2) of the OFs before reconstructing the matrix significantly reduces the computational requirements, resulting in less detailed animations.

Despite the limitations discussed above, the presented TomoFlow method provides a promising new insight into what can be achieved in cryo-ET studies of macromolecular conformational variability. The advancement in OF development might allow even better TomoFlow performance in the future. TomoFlow is not directly applicable to analyzing 2D images. However, it can analyze 3D volumes reconstructed from 2D images, potentially coming from other cryo-EM modalities, such as single-particle images. A TomoFlow application to analyzing continuous conformational variability in single-particle EM maps would require combining with discrete-classification approaches to get the EM maps reconstructed from as conformationally homogeneous groups of single-particle images as possible. In the future, we will work on extending the method to single-particle cryo-EM applications.

\section{Acknowledgments}

We acknowledge the support of the French National Research Agency - ANR (ANR-20-CE11-0020-01 to A.L., ANR-20-CE11-0020-02 to M.E., ANR-20-CE11-0020-03 and ANR-19-CE11-0008-01 to S.J.); German Research Foundation (DFG EL 861/1 to M.E.); the Sorbonne University (2019 "Interface pour le Vivant" PhD scholarship grant to M.H.); and the access to the HPC resources of CINES and IDRIS granted by GENCI (A0100710998, A0070710998, AP010712190, AD011012188 to S.J.). 


\section{Author Contributions}

Mohamad Harastani: Conceptualization, Methodology, Software, Investigation, Validation, WritingOriginal draft preparation. Slavica Jonic: Conceptualization, Methodology, Validation, Writing - Review \& Editing, Project administration, Funding acquisition. Amélie Leforestier: Supervision, Validation, Writing - Review \& Editing, Funding acquisition. Mikhail Eltsov: Supervision, Validation, Writing - Review \& Editing, Funding acquisition.

\section{Data Availability Statement}

The original contributions presented in the study are included in the article/supplementary files. TomoFlow software code will be publicly available on Github (https://github.com/scipion-em/scipionem-continuousflex) and will also be part of the open-source ContinuousFlex plugin of Scipion V3.0. The nucleosome data used in this article are public (available in EMPIAR and EMDB databases under the accession codes EMPIAR-10679 and EMD-12699). The simulated datasets used in this article, with their ground-truth tables, will be made publicly available on Zenodo (DOI: 10.5281/zenodo.XXXXXXX). All questions regarding the software or data availability can be addressed to the corresponding author.

\section{References}

[1] Dobro, M.J., Melanson, L.A., Jensen, G.J., McDowall, A.W. Chapter Three - Plunge Freezing for Electron Cryomicroscopy. In: Jensen GJ, editor. Methods in Enzymology: Academic Press; 2010. p. 63-82. [2] Moor, H. Theory and Practice of High Pressure Freezing. In: Steinbrecht RA, Zierold K, editors. Cryotechniques in Biological Electron Microscopy. Berlin, Heidelberg: Springer Berlin Heidelberg; 1987. p. $175-91$.

[3] Giannuzzi, L.A., Stevie, F.A. (1999). A review of focused ion beam milling techniques for TEM specimen preparation. Micron 30, 197-204.

[4] Ladinsky, M.S., Pierson, J.M., McIntosh, J.R. (2006). Vitreous cryo-sectioning of cells facilitated by a micromanipulator. Journal of Microscopy 224, 129-34. 
[5] Radermacher, M. Weighted Back-projection Methods. In: Frank J, editor. Electron Tomography: Methods for Three-Dimensional Visualization of Structures in the Cell. New York, NY: Springer New York; 2006. p. 245-73.

[6] Jin, Q., Sorzano, Carlos Oscar S., de la Rosa-Trevín, José M., Bilbao-Castro, José R., Núñez-Ramírez, R., Llorca, O., Tama, F., Jonic, S. (2014). Iterative Elastic 3D-to-2D Alignment Method Using Normal Modes for Studying Structural Dynamics of Large Macromolecular Complexes. Structure 22, 496-506.

[7] Dashti, A., Schwander, P., Langlois, R., Fung, R., Li, W., Hosseinizadeh, A., Liao, H.Y., Pallesen, J., Sharma, G., Stupina, V.A., Simon, A.E., Dinman, J.D., Frank, J., Ourmazd, A. (2014). Trajectories of the ribosome as a Brownian nanomachine. Proc Natl Acad Sci U S A 111, 17492-7.

[8] Haselbach, D., Komarov, I., Agafonov, D.E., Hartmuth, K., Graf, B., Dybkov, O., Urlaub, H., Kastner, B., Luhrmann, R., Stark, H. (2018). Structure and Conformational Dynamics of the Human Spliceosomal B(act) Complex. Cell 172, 454-64 e11.

[9] Dashti, A., Mashayekhi, G., Shekhar, M., Ben Hail, D., Salah, S., Schwander, P., des Georges, A., Singharoy, A., Frank, J., Ourmazd, A. (2020). Retrieving functional pathways of biomolecules from singleparticle snapshots. Nat Commun 11, 4734.

[10] Penczek, P.A., Kimmel, M., Spahn, C.M. (2011). Identifying conformational states of macromolecules by eigen-analysis of resampled cryo-EM images. Structure 19, 1582-90.

[11] Katsevich, E., Katsevich, A., Singer, A. (2015). Covariance Matrix Estimation for the Cryo-EM Heterogeneity Problem. SIAM J Imaging Sci 8, 126-85.

[12] Tagare, H.D., Kucukelbir, A., Sigworth, F.J., Wang, H., Rao, M. (2015). Directly reconstructing principal components of heterogeneous particles from cryo-EM images. J Struct Biol 191, 245-62.

[13] Harastani, M., Sorzano, C.O.S., Jonić, S. (2020). Hybrid Electron Microscopy Normal Mode Analysis with Scipion. Protein Science 29, 223-36.

[14] Zhong, E.D., Bepler, T., Berger, B., Davis, J.H. (2021). CryoDRGN: reconstruction of heterogeneous cryo-EM structures using neural networks. Nature Methods 18, 176-85.

[15] Punjani, A., Fleet, D.J. (2021). 3D variability analysis: Resolving continuous flexibility and discrete heterogeneity from single particle cryo-EM. Journal of Structural Biology 213, 107702. 
[16] Giraldo-Barreto, J., Ortiz, S., Thiede, E.H., Palacio-Rodriguez, K., Carpenter, B., Barnett, A.H., Cossio, P. (2021). A Bayesian approach to extracting free-energy profiles from cryo-electron microscopy experiments. Sci Rep 11, 13657.

[17] Eltsov, M., Grewe, D., Lemercier, N., Frangakis, A., Livolant, F., Leforestier, A. (2018). Nucleosome conformational variability in solution and in interphase nuclei evidenced by cryo-electron microscopy of vitreous sections. Nucleic Acids Res 46, 9189-200.

[18] Harastani, M., Eltsov, M., Leforestier, A., Jonic, S. (2021). HEMNMA-3D: Cryo Electron Tomography Method Based on Normal Mode Analysis to Study Continuous Conformational Variability of Macromolecular Complexes. Frontiers in molecular biosciences 8, 663121.

[19] Gubins, I., Chaillet, M.L., van der Schot, G., Veltkamp, R.C., Förster, F., Hao, Y., Wan, X., Cui, X., Zhang, F., Moebel, E., Wang, X., Kihara, D., Zeng, X., Xu, M., Nguyen, N.P., White, T., Bunyak, F. (2020). SHREC 2020: Classification in cryo-electron tomograms. Computers \& Graphics 91, 279-89.

[20] Wan, W., Briggs, J.A.G. Chapter Thirteen - Cryo-Electron Tomography and Subtomogram Averaging. In: Crowther RA, editor. Methods in Enzymology: Academic Press; 2016. p. 329-67.

[21] Scheres, S.H.W., Melero, R., Valle, M., Carazo, J.-M. (2009). Averaging of Electron Subtomograms and Random Conical Tilt Reconstructions through Likelihood Optimization. Structure 17, 1563-72.

[22] Stölken, M., Beck, F., Haller, T., Hegerl, R., Gutsche, I., Carazo, J.-M., Baumeister, W., Scheres, S.H.W., Nickell, S. (2011). Maximum likelihood based classification of electron tomographic data. Journal of Structural Biology 173, 77-85.

[23] Förster, F., Pruggnaller, S., Seybert, A., Frangakis, A.S. (2008). Classification of cryo-electron subtomograms using constrained correlation. Journal of Structural Biology 161, 276-86.

[24] Himes, B.A., Zhang, P. (2018). emClarity: software for high-resolution cryo-electron tomography and subtomogram averaging. Nature Methods 15, 955-61.

[25] Hoppe, W., Gassmann, J., Hunsmann, N., Schramm, H., Sturm, M. (1974). Three-dimensional reconstruction of individual negatively stained yeast fatty-acid synthetase molecules from tilt series in the electron microscope. Hoppe-Seyler's Zeitschrift fur physiologische Chemie 355, 1483-7.

[26] Zhang, L., Ren, G. (2012). High-resolution single-molecule structure revealed by electron microscopy and individual particle electron tomography. J Phys Chem Biophys 2, 10.4172. 
[27] Moebel, E., Kervrann, C. (2020). A Monte Carlo framework for missing wedge restoration and noise removal in cryo-electron tomography. Journal of Structural Biology: X 4, 100013.

[28] Zhai, X., Lei, D., Zhang, M., Liu, J., Wu, H., Yu, Y., Zhang, L., Ren, G. (2020). LoTToR: An Algorithm for Missing-Wedge Correction of the Low-Tilt Tomographic 3D Reconstruction of a Single-Molecule Structure. Scientific Reports 10, 10489.

[29] Wold, S., Esbensen, K., Geladi, P. (1987). Principal component analysis. Chemometrics and Intelligent Laboratory Systems 2, 37-52.

[30] Shah, S.T.H., Xuezhi, X. (2021). Traditional and modern strategies for optical flow: an investigation. SN Applied Sciences 3, 289.

[31] Abrishami, V., Vargas, J., Li, X., Cheng, Y., Marabini, R., Sorzano, C.Ó.S., Carazo, J.M. (2015). Alignment of direct detection device micrographs using a robust Optical Flow approach. Journal of Structural Biology 189, 163-76.

[32] Kazemi, M., Sorzano, C.O.S., Carazo, J.M., Georges, A.d., Abrishami, V., Vargas, J. (2021). ENRICH: A fast method to improve the quality of flexible macromolecular reconstructions. Progress in Biophysics and Molecular Biology 164, 92-100.

[33] Zhou, K., Gaullier, G., Luger, K. (2019). Nucleosome structure and dynamics are coming of age. Nat Struct Mol Biol 26, 3-13.

[34] Armeev, G.A., Kniazeva, A.S., Komarova, G.A., Kirpichnikov, M.P., Shaytan, A.K. (2021). Histone dynamics mediate DNA unwrapping and sliding in nucleosomes. Nat Commun 12, 2387.

[35] Zlatanova, J., Bishop, T.C., Victor, J.M., Jackson, V., van Holde, K. (2009). The nucleosome family: dynamic and growing. Structure 17, 160-71.

[36] Bilokapic, S., Strauss, M., Halic, M. (2018). Histone octamer rearranges to adapt to DNA unwrapping. Nat Struct Mol Biol 25, 101-8.

[37] Koopmans, W.J., Buning, R., Schmidt, T., van Noort, J. (2009). spFRET using alternating excitation and FCS reveals progressive DNA unwrapping in nucleosomes. Biophys J 97, 195-204.

[38] Ngo, T.T., Ha, T. (2015). Nucleosomes undergo slow spontaneous gaping. Nucleic Acids Res 43, 396471.

[39] Cai, S., Bock, D., Pilhofer, M., Gan, L. (2018). The in situ structures of mono-, di-, and trinucleosomes in human heterochromatin. Mol Biol Cell 29, 2450-7. 
[40] Farnebäck, G. Two-Frame Motion Estimation Based on Polynomial Expansion. In: Bigun J, Gustavsson T, editors. Image Analysis. Berlin, Heidelberg: Springer Berlin Heidelberg; 2003. p. 363-70. [41] Lucas, B.D., Kanade, T. An iterative image registration technique with an application to stereo vision. Vancouver, British Columbia; 1981.

[42] Bouguet, J.-Y. (2001). Pyramidal implementation of the affine lucas kanade feature tracker description of the algorithm. Intel corporation 5, 4.

[43] Kunz, M., Frangakis, A.S. (2017). Three-dimensional CTF correction improves the resolution of electron tomograms. Journal of Structural Biology 197, 114-22.

[44] Turoňová, B., Schur, F.K.M., Wan, W., Briggs, J.A.G. (2017). Efficient 3D-CTF correction for cryoelectron tomography using NovaCTF improves subtomogram averaging resolution to $3.4 \AA$. Journal of Structural Biology 199, 187-95.

[45] Singla, J., White, K.L., Stevens, R.C., Alber, F. (2021). Assessment of scoring functions to rank the quality of 3D subtomogram clusters from cryo-electron tomography. Journal of Structural Biology 213, 107727.

[46] Chen, M., Bell, J.M., Shi, X., Sun, S.Y., Wang, Z., Ludtke, S.J. (2019). A complete data processing workflow for cryo-ET and subtomogram averaging. Nature Methods 16, 1161-8.

[47] Bharat, T.A.M., Scheres, S.H.W. (2016). Resolving macromolecular structures from electron cryotomography data using subtomogram averaging in RELION. Nature Protocols 11, 2054-65.

[48] Navarro, P.P., Stahlberg, H., Castaño-Díez, D. (2018). Protocols for Subtomogram Averaging of Membrane Proteins in the Dynamo Software Package. Frontiers in molecular biosciences 5, 82-.

[49] Chen, Y., Pfeffer, S., Hrabe, T., Schuller, J.M., Förster, F. (2013). Fast and accurate reference-free alignment of subtomograms. Journal of Structural Biology 182, 235-45.

[50] Slabaugh, G.G. (1999). Computing Euler angles from a rotation matrix. Retrieved on August 6, 3963.

[51] Xu, M., Beck, M., Alber, F. (2012). High-throughput subtomogram alignment and classification by Fourier space constrained fast volumetric matching. Journal of Structural Biology 178, 152-64.

[52] Ma, J. (2005). Usefulness and Limitations of Normal Mode Analysis in Modeling Dynamics of Biomolecular Complexes. Structure 13, 373-80. 
[53] Tama, F., Miyashita, O., Brooks, C.L. (2004). Flexible Multi-scale Fitting of Atomic Structures into Low-resolution Electron Density Maps with Elastic Network Normal Mode Analysis. Journal of Molecular Biology 337, 985-99.

[54] Frenkel, D., Smit, B. Understanding molecular simulation: from algorithms to applications: Elsevier; 2001.

[55] Kobayashi, C., Jung, J., Matsunaga, Y., Mori, T., Ando, T., Tamura, K., Kamiya, M., Sugita, Y. GENESIS 1.1: A hybrid-parallel molecular dynamics simulator with enhanced sampling algorithms on multiple computational platforms. Wiley Online Library; 2017. p. 2193-206.

[56] Peng, L.-M., Ren, G., Dudarev, S., Whelan, M. (1996). Robust parameterization of elastic and absorptive electron atomic scattering factors. Acta Crystallographica Section A: Foundations of Crystallography 52, 257-76.

[57] Sorzano, C.O., de la Rosa Trevín, J.M., Otón, J., Vega, J.J., Cuenca, J., Zaldívar-Peraza, A., GómezBlanco, J., Vargas, J., Quintana, A., Marabini, R., Carazo, J.M. Semiautomatic, High-Throughput, HighResolution Protocol for Three-Dimensional Reconstruction of Single Particles in Electron Microscopy. In: Sousa AA, Kruhlak MJ, editors. Nanoimaging: Methods and Protocols. Totowa, NJ: Humana Press; 2013. p. 171-93.

[58] Kunz, M., Frangakis, A.S. (2014). Super-sampling SART with ordered subsets. J Struct Biol 188, 10715.

[59] de la Rosa-Trevín, J.M., Quintana, A., del Cano, L., Zaldívar, A., Foche, I., Gutiérrez, J., Gómez-Blanco, J., Burguet-Castell, J., Cuenca-Alba, J., Abrishami, V., Vargas, J., Otón, J., Sharov, G., Vilas, J.L., Navas, J., Conesa, P., Kazemi, M., Marabini, R., Sorzano, C.O.S., Carazo, J.M. (2016). Scipion: A software framework toward integration, reproducibility and validation in 3D electron microscopy. Journal of Structural Biology 195, 93-9.

[60] Strelak, D., Jimenez-Moreno, A., Vilas, J.L., Ramirez-Aportela, E., Sanchez-Garcia, R., Maluenda, D., Vargas, J., Herreros, D., Fernandez-Gimenez, E., de Isidro-Gomez, F.P., Horacek, J., Myska, D., Horacek, M., Conesa, P., Fonseca-Reyna, Y.C., Jimenez, J., Martinez, M., Harastani, M., Jonic, S., Filipovic, J., Marabini, R., Carazo, J.M., Sorzano, C.O.S. (2021). Advances in Xmipp for Cryo-Electron Microscopy: From Xmipp to Scipion. Molecules 26. 
[61] Tegunov, D., Xue, L., Dienemann, C., Cramer, P., Mahamid, J. (2021). Multi-particle cryo-EM refinement with $\mathrm{M}$ visualizes ribosome-antibiotic complex at $3.5 \mathrm{~A}$ in cells. Nat Methods 18, 186-93. [62] Dong, H., Qin, S., Zhou, H.X. (2010). Effects of macromolecular crowding on protein conformational changes. PLoS Comput Biol 6, e1000833. 
This is the Supplementary Material of an article accepted for publication in Journal of

Molecular Biology, doi: https://doi.org/10.1016/j.jmb.2021.167381

\title{
TomoFlow: Analysis of continuous conformational variability of macromolecules in cryogenic subtomograms based on 3D dense optical flow
}

\author{
Mohamad Harastani ${ }^{1,2}$, Mikhail Eltsov ${ }^{3}$, Amélie Leforestier $^{2}$, and Slavica Jonic ${ }^{1,}{ }^{*}$ \\ ${ }^{1}$ IMPMC - UMR 7590 CNRS, Sorbonne Université, Muséum National d'Histoire Naturelle, Paris, France \\ ${ }^{2}$ Laboratoire de Physique des Solides (LPS), UMR 8502 CNRS, Université Paris-Saclay, Orsay, France \\ ${ }^{3}$ Department of Integrated Structural Biology, Institute of Genetics and Molecular and Cellular Biology, Illkirch, France
}

Contact details of the corresponding author:

Dr. Slavica Jonić

Sorbonne Université

IMPMC - CNRS UMR 7590, CC 115

4 Place Jussieu, 75005 Paris, France

Tel: +33144277205

Fax: +33144273785

E-mail: slavica.jonic@upmc.fr 


\section{Supplementary Method Description 1:}

\section{Multiresolution pyramidal approach for 3D optical flow calculation}

In this section, we describe the multiresolution pyramidal approach for 3D optical flow (OF) calculation with Farneback-3D toolbox (https://pypi.org/project/farneback3d), which is used in TomoFlow.

The 3D OF pyramidal approach involves (i) creating a multiresolution volume pyramid by downsampling the volume at each pyramid level (Supplementary Figure 1), (ii) calculating OF iteratively at each pyramid level, and (iii) propagating the OF calculated at a coarser level to the next finer level in order to refine it, until the finest (original volume) level is reached. Between the pyramid scales, the OF propagation is done by upsampling the OF found on a coarser level to the next finer level and applying this upsampled OF onto the reference volume at the finer level to create a warped reference that is then used to find the OF at that finer level.

In Farneback-3D, an anti-aliasing Gaussian filtering is applied onto the volume at each pyramid level before the volume is downsampled (the Gaussian standard deviation is adjusted to the scaling factor selected for downsampling). The scaling factor of 0.5 was used in the experiments in this article, meaning that each volume dimension was reduced by 2 at each pyramid level. In the experiments in this article, the coarsest volume pyramid level is $32 \times 32 \times 32$ voxels, which is the coarsest level allowed by Farneback-3D; also, we used 2-level pyramids for volumes of size $64^{3}$ voxels and 3 -level pyramids for volumes of $128^{3}$ voxels. We used a window size of $10 \times$ $10 \times 10$ voxels for integrating the displacement field over a neighborhood of each voxel and 10 iterations of the algorithm at each pyramid level. All other parameters of Farneback-3D were used with their default values. TomoFlow graphical interface allows modifying these values.

The OF is first calculated on the coarsest pyramid level (lowest scale) and, then, it is refined on the first finer pyramid level (larger scale), followed by the refinement on the next one etc., until the refinement on the finest pyramid level (original scale, i.e., the input volumes). For each pyramid level, the OF is calculated iteratively. In each iteration, the calculated OF is applied onto the reference volume to warp it; this warped reference is then used to find the OF in the next iteration and produce the reference for the following iteration, etc., until the convergence is achieved (the OF between two successive iterations does not change significantly).

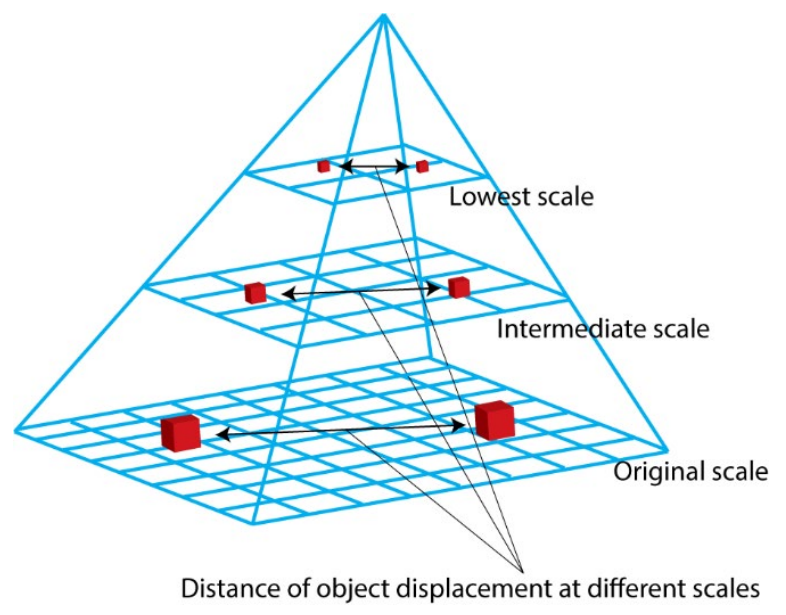

Supplementary Figure 1: Multiresolution data pyramid scheme. 


\section{Supplementary Experiment 1:}

\section{Robustness to noise while matching different magnitudes of displacements, disentangling conformational mapping from the subtomographic-approach limitations}

In this section, we show the performance of the Farneback 3D optical flow (OF) method in matching different conformational variability magnitudes, challenged by noise only. Also, we provide a quantitative assessment of the algorithm for mapping conformations while disentangling it from the subtomographic-approach limitations such as missing wedge and rigid-body (angular and shift) variability. The PDB:4AKE chain A structure (obtained at $2.2 \AA$ resolution by X-ray crystallography and referred here to as AK) was used to synthesize three conformations by elastic deforming AK using its normal mode 7 and gradually increasing the amplitude of the mode. The three synthesized conformations were then converted into volumes (volume size: $128^{3}$ voxels; voxel size: $1 \AA^{3}$ ) and noise was applied directly onto these volumes (without low-pass filtering of the volumes or synthesizing tilt series and calculating $3 \mathrm{D}$ reconstructions).

The conformational distance of each of the three synthetic conformations is reflected by the selected amplitude of normal mode 7. The following three values of the amplitude were used: 1) -75 (the structure referred to as AK_75), 2) -125 (the structure referred to as AK_125), and 3) -200 (the structure referred to as AK_200). The four atomic structures (AK, AK_75, AK_125 and AK_200) are shown in Supplementary Figure 2. These structures converted into volumes are shown in Supplementary Figure 3.

The root mean square deviations (RMSDs) of the AK_75, AK_125 and AK_200 structures with respect to the AK structure are shown in Supplementary Table 1, along with the cross-correlations (CC) between the AK volume and each of the AK_75, AK_125 and AK_200 volumes.

Random Gaussian noise was added to each of the AK_75, AK_125 and AK_200 volumes in such a way to obtain the following 6 values of the signal-to-noise ratio (SNR): 1) $0.5,2) 0.1,3$ ) $0.05,4) 0.01,5) 0.005$, and 6) 0.001 . In Supplementary Figure 4, we show the different SNR values of the volumes using central slices of the noisy AK_125 volumes as an example.

The OF was calculated using Farneback-3D with a 3-level volume pyramid of scaling factor of 0.5 (meaning a pyramid with the levels of $128^{3}, 64^{3}$ and $32^{3}$ voxels for the test datasets analyzed in this section, where $32^{3}$ voxels is the coarsest pyramid level allowed by Farneback-3D).

We calculated the OFs between the non-noisy AK volume and the noisy AK_75, AK_125 and AK_200 volumes (6 SNR values for each of AK_75, AK_125 and AK_200). Each OF was used to warp the AK volume. The obtained warped AK volumes are the non-noisy estimates of the noisy AK_75, AK_125 and AK_200 volumes and are called "matched" volumes (the term introduced in the main text of the article).

The "matched" volumes for AK_75, AK_125 and AK_200 are shown in Supplementary Figures 5, 6 and 7 respectively. The CCs between the non-noisy versions of each of the AK_75, AK_125 and AK_200 volumes and the corresponding "matched" volumes are presented in Supplementary Table 2.

The visual comparison in Supplementary Figures 5-7 and the corresponding results in Supplementary Table 2 indicate that the "matched" volume approached the conformation in all different noisy volumes (the CC between the "matched" volume and each of the non-noisy versions of the AK_75, AK_125 and AK_200 volumes is always higher than the original cross correlation before the matching). Better results were obtained for smaller magnitudes of the conformational change and lower noise levels (Supplementary Table 2). 


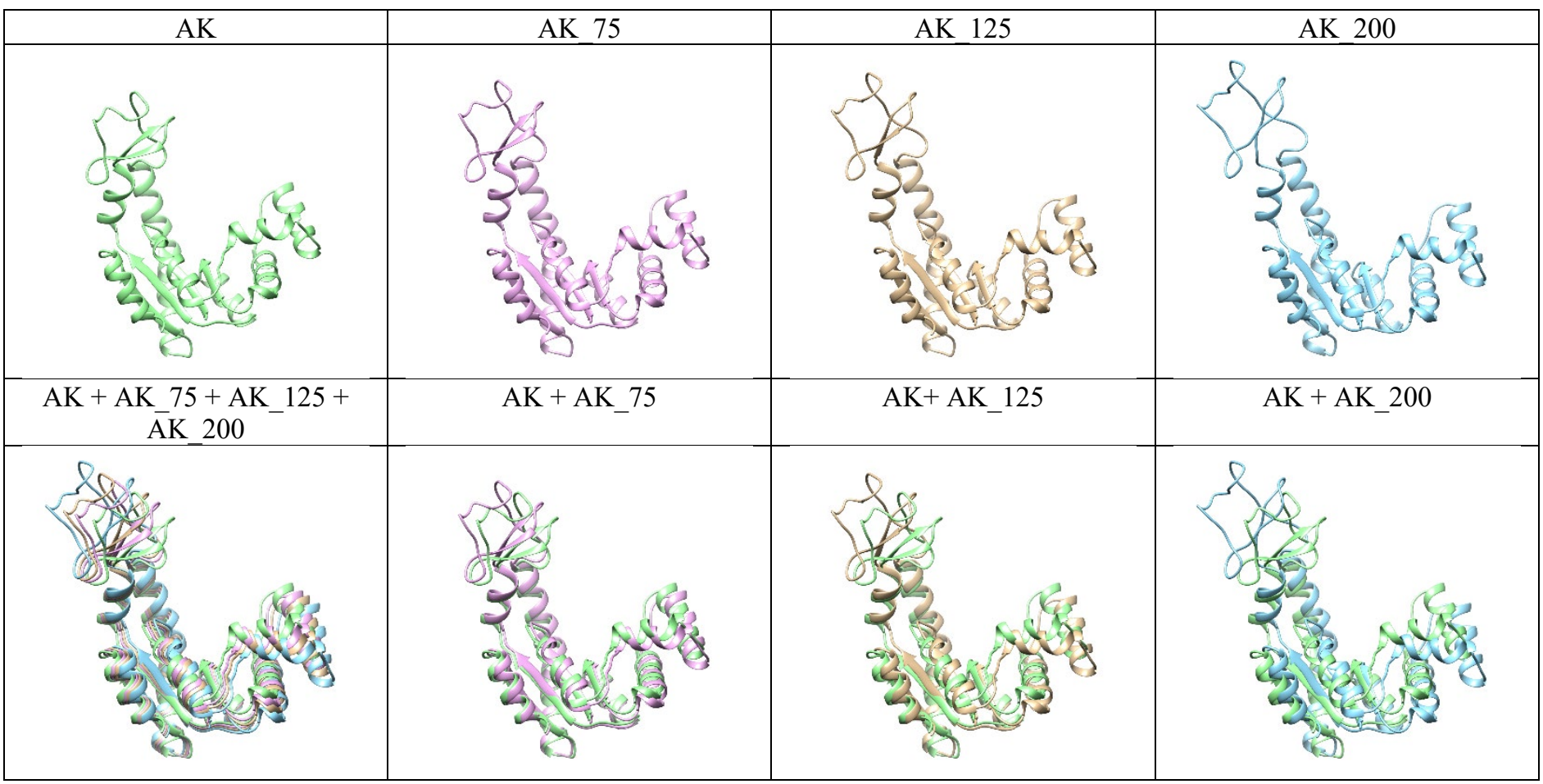

Supplementary Figure 2: Atomic structures used in the experiment. See the text in this section for details on how they were obtained.

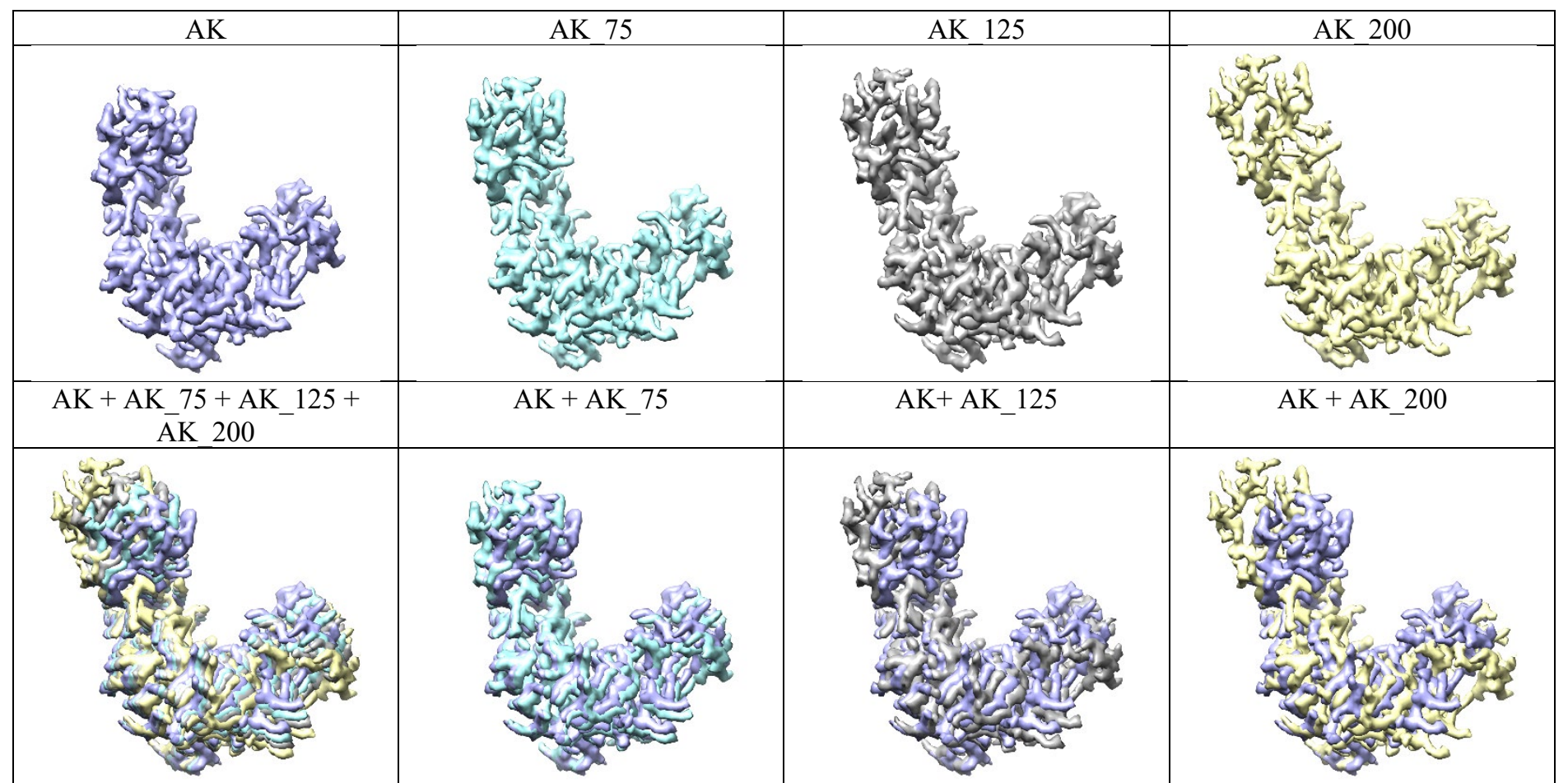

Supplementary Figure 3: Volumes used in the experiment. See the text in this section for details on how they were obtained. 


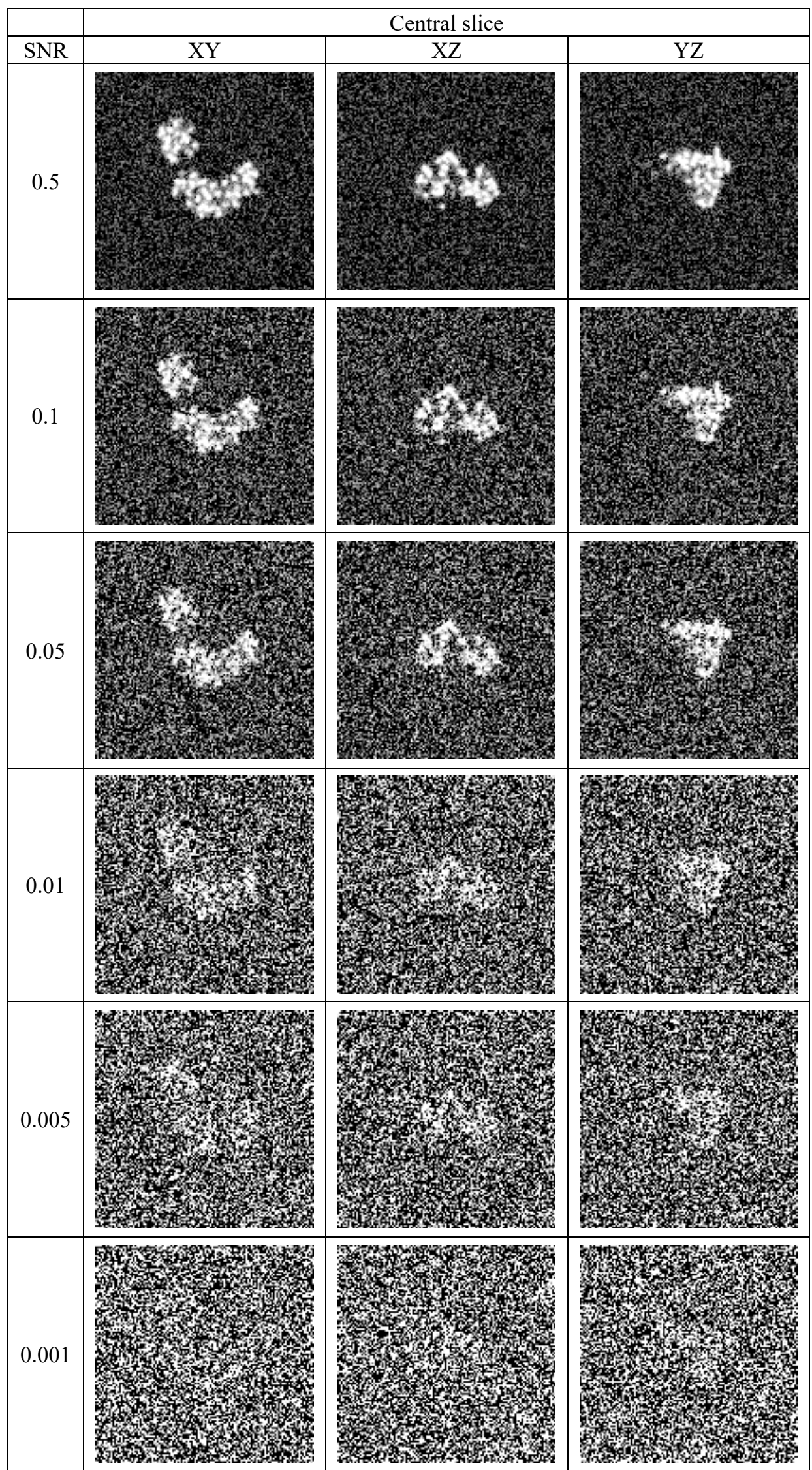

Supplementary Figure 4: Central slices of the volume AK125 at different values of the signal-to-noise ratio (SNR). 


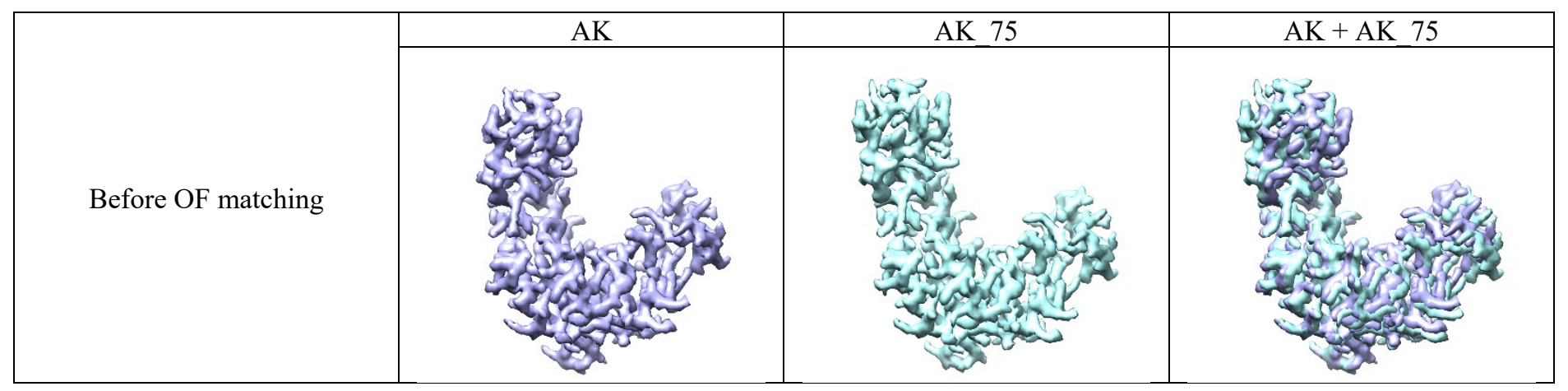

\begin{tabular}{|c|c|c|c|}
\hline \multicolumn{4}{|c|}{ After OF matching } \\
\hline Matched SNR 0.5 & Matched SNR $0.5+$ AK 75 & Matched SNR 0.1 & Matched SNR 0.1 + AK 75 \\
\hline 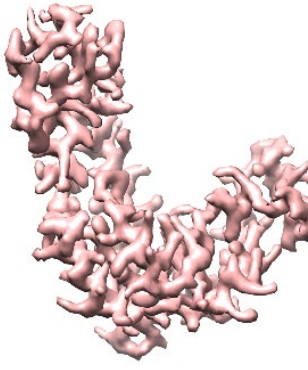 & 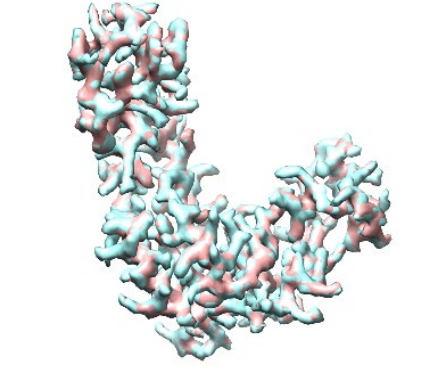 & 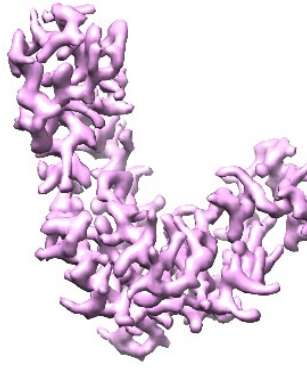 & 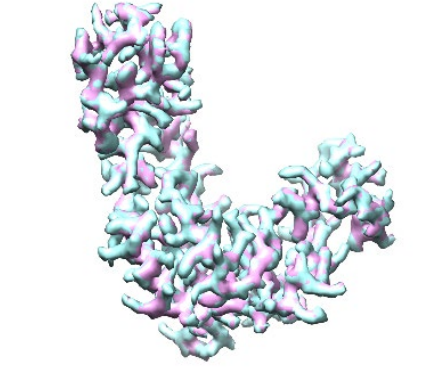 \\
\hline Matched SNR 0.05 & Matched SNR $0.05+$ AK_75 & Matched SNR 0.01 & Matched SNR $0.01+$ AK 75 \\
\hline 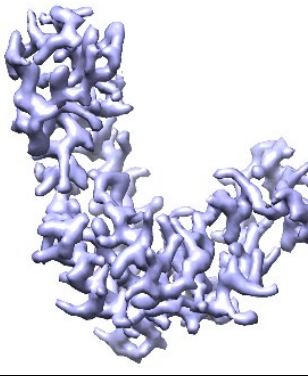 & 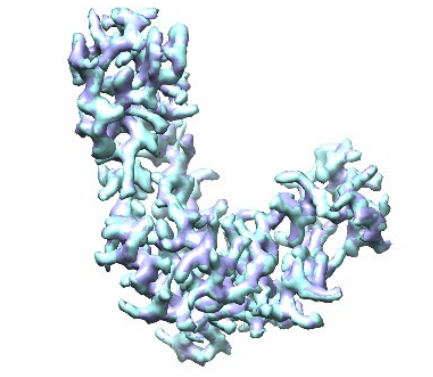 & 5 & 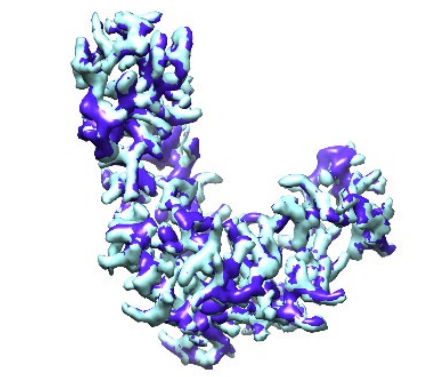 \\
\hline Matched SNR 0.005 & Matched SNR $0.005+$ AK_75 & Matched SNR 0.001 & Matched SNR 0.001 + AK_75 \\
\hline 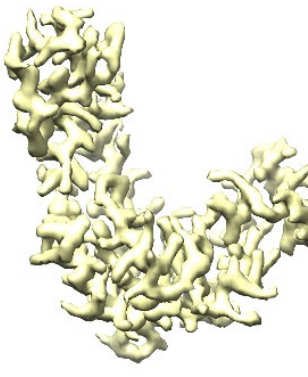 & 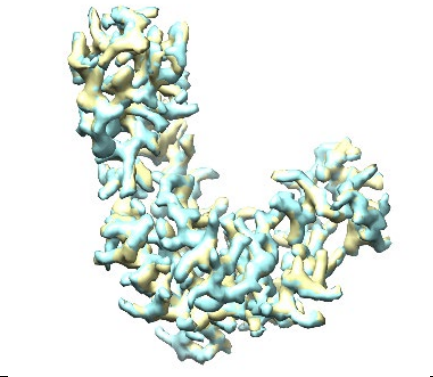 & 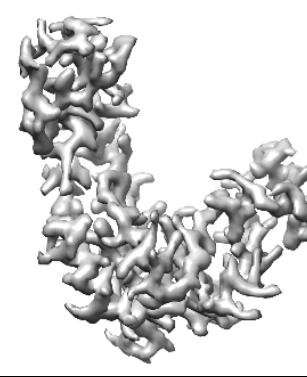 & 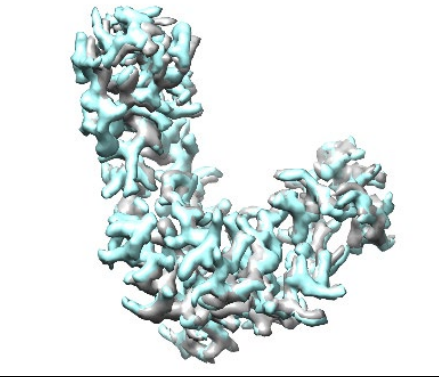 \\
\hline
\end{tabular}

Supplementary Figure 5: OF-based matching of the non-noisy AK volume to different noisy versions of the AK_75 volume. 


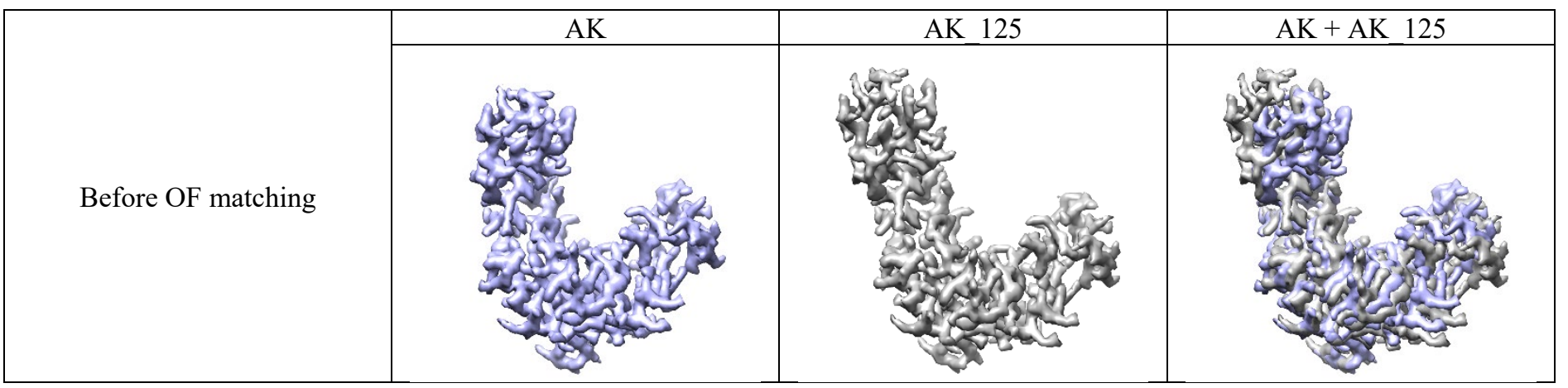

\begin{tabular}{|c|c|c|c|}
\hline \multicolumn{4}{|c|}{ After OF matching } \\
\hline Matched SNR 0.5 & Matched SNR $0.5+$ AK 125 & Matched SNR 0.1 & Matched SNR 0.1 + AK_ 125 \\
\hline 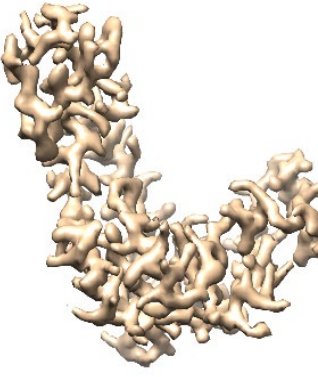 & 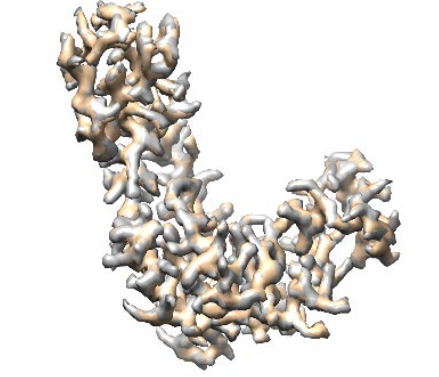 & 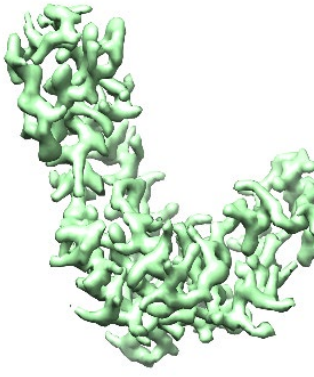 & 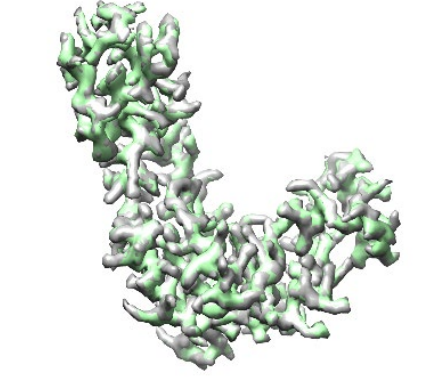 \\
\hline Matched SNR 0.05 & Matched SNR $0.05+$ AK_125 & Matched SNR 0.01 & Matched SNR $0.01+$ AK_125 \\
\hline 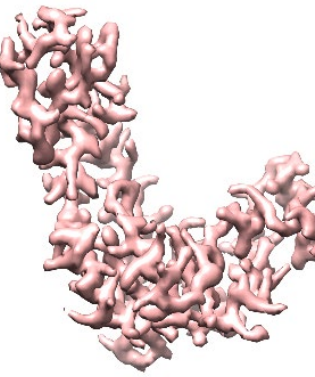 & 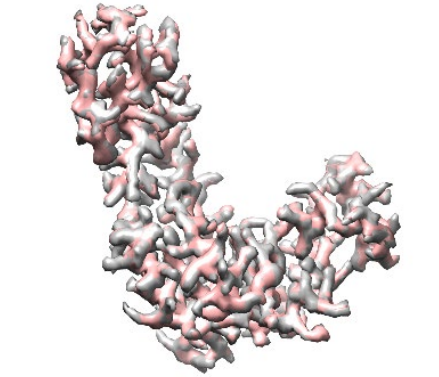 & 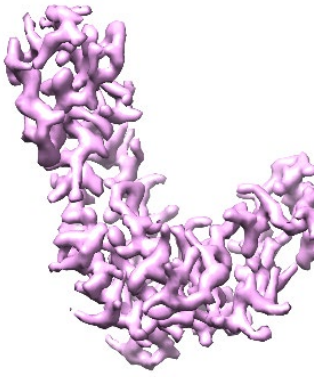 & 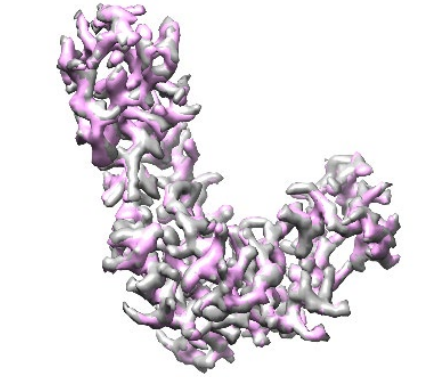 \\
\hline Matched SNR 0.005 & $\begin{array}{c}\text { Matched SNR } 0.005+ \\
\text { AK_125 } \\
\end{array}$ & Matched SNR 0.001 & $\begin{array}{c}\text { Matched SNR } 0.001+ \\
\text { AK_125 } \\
\end{array}$ \\
\hline 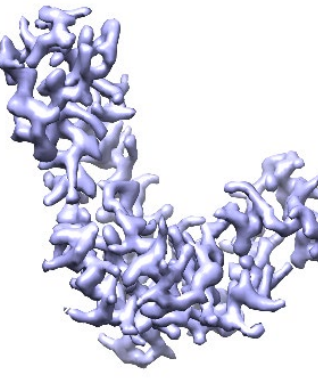 & 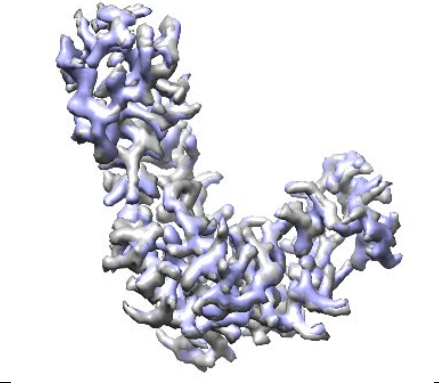 & 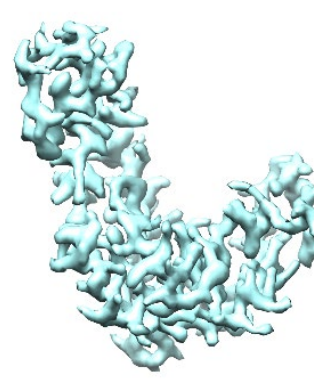 & 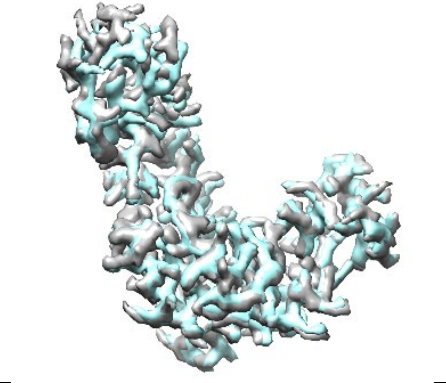 \\
\hline
\end{tabular}

Supplementary Figure 6: OF-based matching of the non-noisy AK volume to different noisy versions of the AK_125 volume. 


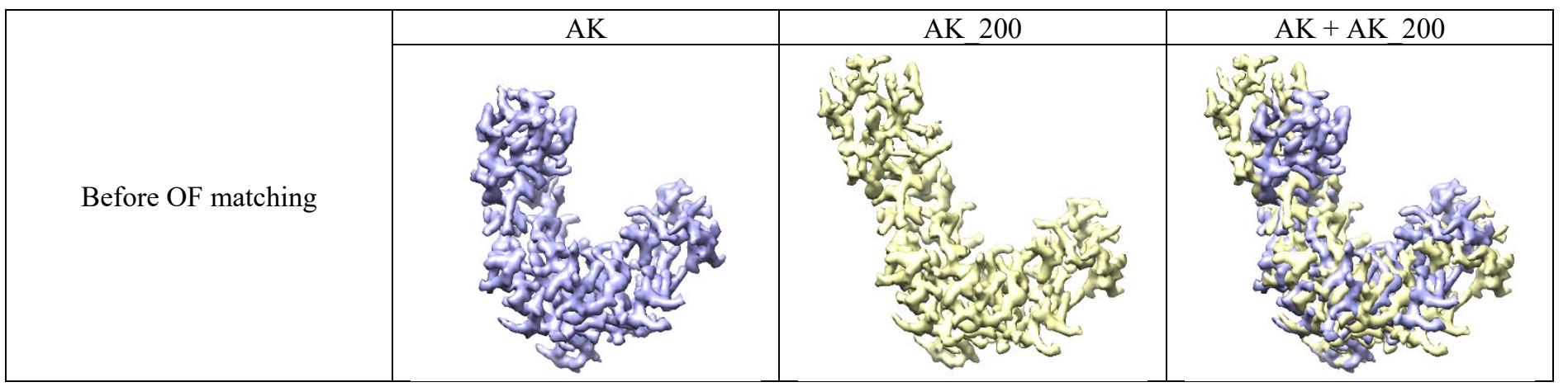

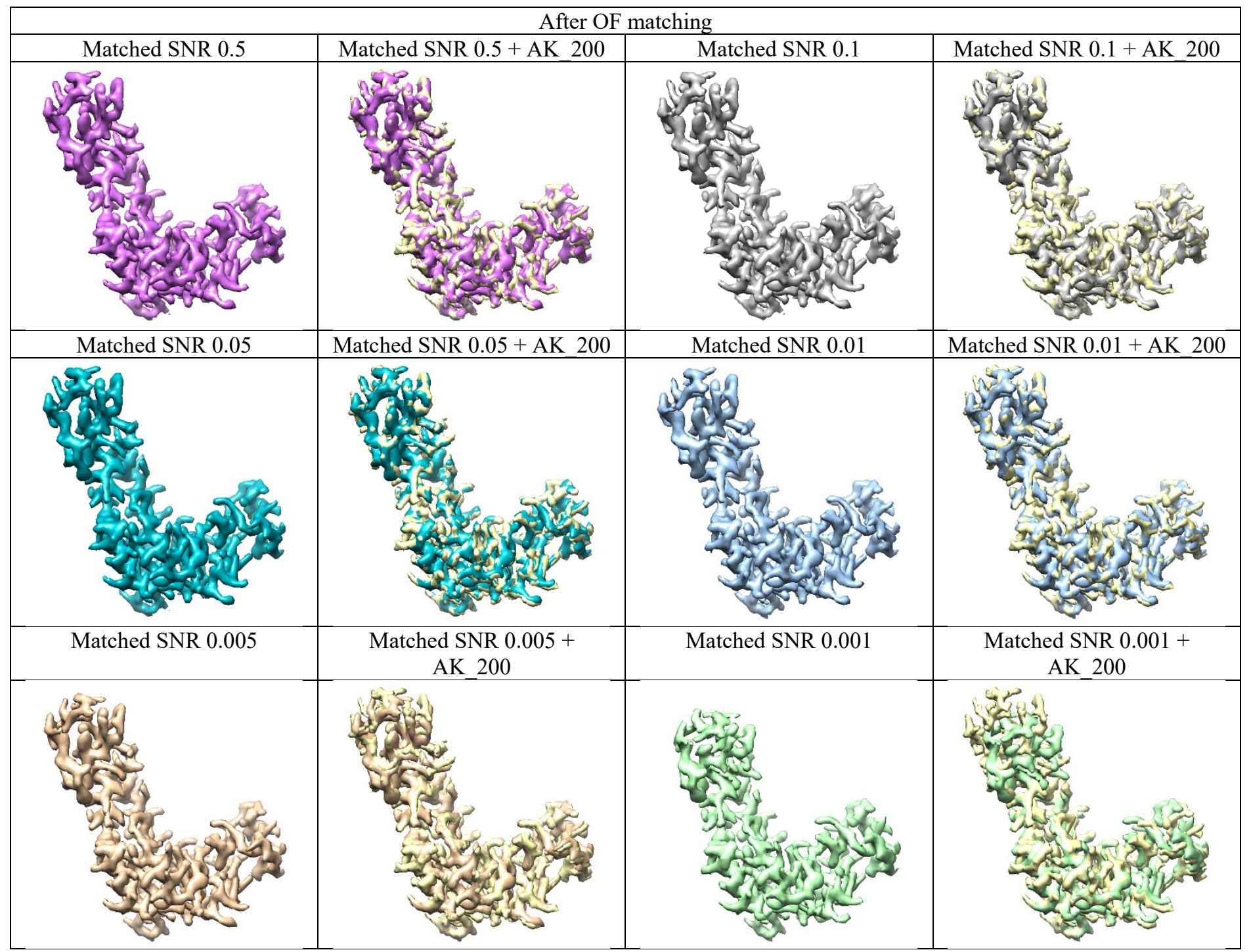

Supplementary Figure 7: OF-based matching of the non-noisy AK volume to different noisy versions of the AK_200 volume. 


\begin{tabular}{|c|c|c|c|}
\hline & AK 75 & AK 125 & AK 200 \\
\hline RMSD compared to AK $[\AA]$ & 1.8431 & 3.0719 & 4.915 \\
\hline CC with AK & $83.78 \%$ & $73.27 \%$ & $61.59 \%$ \\
\hline
\end{tabular}

Supplementary Table 1: Quantitively measure of the difference between the AK conformation and each of the three synthetic conformations used here (AK_75, AK_125 and AK_200), expressed in terms of the root mean square deviation (RMDS) between the atomic structures and in terms of the cross-correlation (CC) between the volumes from these atomic structures. Note here that the $\mathrm{CC}$ in this table is calculated for non-noisy volumes.

\begin{tabular}{|c|c|c|c|c|c|c|}
\hline & SNR 0.001 & SNR 0.005 & SNR 0.01 & SNR 0.05 & SNR 0.1 & SNR 0.5 \\
\hline AK_75 & $94.75 \%$ & $98.18 \%$ & $98.50 \%$ & $98.96 \%$ & $99.01 \%$ & $99.06 \%$ \\
\hline AK_125 & $92.71 \%$ & $97.23 \%$ & $97.90 \%$ & $98.52 \%$ & $98.65 \%$ & $98.65 \%$ \\
\hline AK_200 & $86.23 \%$ & $95.27 \%$ & $96.56 \%$ & $97.46 \%$ & $97.53 \%$ & $97.63 \%$ \\
\hline
\end{tabular}

Supplementary Table 2: Cross-correlation between the "matched" AK volume and the non-noisy AK_75, AK_125 and AK_200 volumes. The AK volume "matching" was done with respect to different noisy versions of the AK_75, AK_125 and AK_200 volumes. 